\title{
A mirror theorem for toric complete intersections
}

\author{
Alexander Givental *
}

January 27, 1997

\begin{abstract}
We prove a generalized mirror conjecture for non-negative complete intersections in symplectic toric manifolds. Namely, we express solutions of the PDE system describing quantum cohomology of such a manifold in terms of suitable hypergeometric functions.
\end{abstract}

0. Introduction. Let $X$ denote a non-singular compact Kähler toric variety with the Picard number $k$. The variety $X$ can be obtained by the symplectic reduction of the standard Hermitian space $\mathbb{C}^{N}$ by the action of a subtorus $T^{k}$ in the torus $T^{N}$ of diagonal unitary matrices on a generic level of the momentum map (see Section 3). The coordinate hyperplanes in $\mathbb{C}^{N}$ are $T^{N}$-invariant, and their $T^{k}$-reductions on the same level of the momentum map define $N$ compact toric hypersurfaces in $X$. We denote $u_{1}, \ldots, u_{N}$ the classes in $H^{2}(X)$ Poincare-dual to the fundamental cycles of these hypersurfaces. It is known that $H^{2}(X)$ is a free abelian group of rank $k$ spanned by $u_{1}, \ldots, u_{N}$, that it multiplicatively generates the ring $H^{*}(X)$, and that the 1-st Chern class $c\left(\mathcal{T}_{X}\right)$ of the tangent bundle to $X$ is equal to $u_{1}+\ldots+u_{N}$.

Let us consider the sum $\mathcal{V}$ of $l \geq 0$ non-negative line bundles over $X$ with the 1 -st Chern classes $v_{1}, \ldots, v_{l}$ and denote $Y$ the non-singular complete intersection in $X$ of dimension $N-k-l$ defined by global holomorphic sections of these line bundles. The inclusion $Y \subset X$ induces the homomorphism

* Research is partially supported by Alfred P. Sloan Foundation and by NSF grant DMS-9321915 
$H^{2}(X) \rightarrow H^{2}(Y)$ The cohomology ring $H^{*}(Y)$ contains the subring multiplicatively generated by the classes $u_{1}, \ldots, u_{N}$. We denote this subring $H^{*}(\mathcal{V})$. It carries the Poincare pairing $\langle f, g\rangle=\int_{[Y]} f g=\int_{[X]} f g v_{1} \ldots v_{l}$ non-degenerate over $\mathbb{Q}$. The 1 -st Chern class $c\left(\mathcal{T}_{Y}\right)$ of the tangent bundle to $Y$ is equal to $u_{1}+\ldots+u_{N}-v_{1}-\ldots-v_{l}$.

Given a compact Kähler manifold $Y$, the Gromov - Witten theory [14 associates to it the quantum cohomology algebra and the quantum cohomology $\mathcal{D}$-module.

Let us introduce the semigroup $\Lambda \subset H_{2}(Y)$ of fundamental classes of compact holomorphic curves in $Y$. The quantum cohomology algebra is a skew - commutative associative deformation of the cohomology algebra $H^{*}(Y, \mathbb{Q}[[\Lambda]])$ with coefficients in a formal completion of the semigroup algebra $\mathbb{Q}[\Lambda]$. The structural constants $\langle a \circ b, c\rangle$ of the quantum multiplication $\circ$ are formal series $\sum_{d \in \Lambda} n_{d}(a, b, c) q^{d}$ where $q^{d}$ is the element of the semigroup ring corresponding to the homology class $d \in \Lambda$. The coefficient $n_{d}(a, b, c)$ has the meaning of the number of holomorphic maps $\mathbb{C} P^{1} \rightarrow Y$ representing the homology class $d$ and sending $0,1, \infty \in \mathbb{C} P^{1}$ to given cycles in $Y$ Poincaredual to the classes $a, b, c \in H^{*}(Y)$ respectively. We refer to [5, 14] for variants of actual definitions which employ intersection theory in Kontsevich's compactifications of moduli spaces of stable maps $\mathbb{C} P^{1} \rightarrow Y$ (see Section 1). In particular $n_{d}(a, b, c)=0$ unless the total degree of the classes $a, b, c$ equals the real dimension of the fundamental class in the space of maps $\mathbb{C} P^{1} \rightarrow Y$ representing the class $d$. This gives rise to the grading $\operatorname{deg} q^{d}=2 \int_{[d]} c\left(\mathcal{T}_{Y}\right)$ in the quantum cohomology algebra.

In addition to the associativity identity the structural constants of the quantum multiplication satisfy some integrability condition which can be formulated as compatibility of certain system of linear differential equations. Let $\left(t_{1}, \ldots, t_{k}\right)$ denote coordinated on $H^{2}(Y)$ with respect to a basis $\left(p_{1}, \ldots, p_{k}\right)$ of integral symplectic classes. The basis $q^{d}$ in the semigroup ring $\mathbb{Z}[\Lambda]$ can be identified with $q_{1}^{d_{1}} \ldots q_{k}^{d_{k}}=\exp \left(t_{1} d_{1}+\ldots+t_{k} d_{k}\right)$ where $\left(d_{1}, \ldots, d_{k}\right)$ are coordinates of $d \in H_{2}(Y)$ with respect to the dual basis. Denote $t_{0}$ the coordinate on $H^{0}(Y)$. The operators $p_{i}$ o of quantum multiplication act on vector functions $s\left(t_{0}, t\right)$ with values in $H^{*}(Y, \mathbb{C})$. In these notations the integrability condition reads:

$$
\hbar \frac{\partial}{\partial t_{0}} s=s, \hbar \frac{\partial}{\partial t_{i}} s=p_{i} \circ s, i=1, \ldots, k,
$$


form a consistent system of linear PDEs.

Solutions $s$ to the PDE system can also be interpreted in terms of intersection theory on spaces of stable maps (see Section 1 ). The $\mathcal{D}$-module corresponding to this syslem is generated by a single formal vector-function $J_{Y}\left(t, \hbar^{-1}\right)$ with coefficients in $H^{*}(Y, \mathbb{Q})$ (see Section 1 ). It has the following property [10]: whenever a scalar differntial operator $D(\hbar \partial / \partial t, \exp t, \hbar)$ with coefficients in $\mathbb{C}[\hbar][[\Lambda]]$ annihilates (all componenets of the vector-function) $J_{Y}$, the symbol $D(p \circ, q, 0)$ vanishes in the quantum cohomology algebra of $Y$.

A construction of $J_{Y}$ in terms of intersection theory on moduli spaces of stable maps is given in Section 1. By the very definition the function $J_{Y}$ has the the asymptotical expansion

$$
J_{Y}=e^{\left(t_{0}+p_{1} t_{1}+\ldots p_{k} t_{k}\right) / \hbar}(1+o(1 / \hbar)) .
$$

In applications to a toric complete intersection $Y \subset X$ we will detect degrees of holomorphic curves in $Y$ by their degrees in $X$. This means that $\Lambda$ will denote the semigroup of classes of compact holomorphic curves in $X$, $t_{1}, \ldots, t_{k}$ will be coordinates on $H_{2}(X)$, and $J_{Y}$ - a formal series in $q_{i}=e^{t_{i}}$. Notice that in the case where $v_{1}, \ldots, v_{l}$ are positive and $\operatorname{dim} Y>2$, the map $H_{2}(Y) \rightarrow H_{2}(X)$ is an isomorphism and thus the notation $J_{Y}$ has the same meaning as before. However for semi-positive $v_{1}, \ldots, v_{l}$ (and $\operatorname{dim} Y>1$ ) the map $H_{2}(Y) \rightarrow H_{2}(X)$ can have a non-trivial kernel, and therefore $J_{Y}$ is obtained from the vector-function discussed in the previous paragraphs by the restriction operation. [

We will denote $J$ the orthogonal projection of the vector-function $J_{Y}$ to the subalgebra $H^{*}(\mathcal{V}, \mathbb{Q}) \subset H^{*}(Y, \mathbb{Q})$.

Introduce the formal vector-function $I\left(t, \hbar^{-1}\right)$ with values in $H^{*}(\mathcal{V}, \mathbb{Q})$ :

$$
\begin{gathered}
\text { (*) } I=e^{\left(t_{0}+p_{1} t_{1}+\ldots+p_{k} t_{k}\right) / \hbar} \sum_{d \in \Lambda} e^{t_{1} d_{1}+\ldots+t_{k} d_{k}} \times \\
\times \frac{\prod_{a=1}^{l} \Pi_{m=-\infty}^{L_{a}(d)}\left(v_{a}+m \hbar\right) \Pi_{j=1}^{N} \Pi_{m=-\infty}^{0}\left(u_{j}+m \hbar\right)}{\Pi_{a=1}^{l} \Pi_{m=-\infty}^{0}\left(v_{a}+m \hbar\right) \Pi_{\alpha=1}^{N} \Pi_{m=-\infty}^{D_{j}(d)}\left(u_{j}+m \hbar\right)},
\end{gathered}
$$

\footnotetext{
${ }^{1}$ I would like to thank V. Batyrev and B. Kim who pointed me to this subtlety in the semi-positive case.
} 
where $D_{j}(d)=\int_{[d]} u_{j}, L_{a}(d)=\int_{[d]} v_{a}$. The function $I$ is the product of $e^{\left(t_{0}+p \log q\right) / \hbar}$ with a power series in $q=e^{t}$ supported in $\Lambda$ and weightedhomogeneous of degree 0 with respect to the grading

$$
\operatorname{deg} \hbar=\operatorname{deg} u_{j}=\operatorname{deg} v_{a}=1, \operatorname{deg} q^{d}=\int_{[d]} c\left(\mathcal{T}_{Y}\right)
$$

The function $J$ has the same properties (see Section 1).

Theorem 0.1. Let $Y$ be a non-singular toric complete intersection with the non-negative 1-st Chern class $c\left(\mathcal{T}_{Y}\right)$. Then the formal vector-functions $I$ and $J$ with coefficients in $H^{*}(\mathcal{V}, \mathbb{Q})$ coincide up to a triangular weightedhomogeneous change of variables:

$$
t_{0} \mapsto t_{0}+f_{0}(q) \hbar+h(q), \log q_{i} \mapsto \log q_{i}+f_{i}(q)
$$

where $h, f_{0}, f_{1}, \ldots, f_{k}$ are weighted-homogeneous power series supported in $\Lambda$ 0 and $\operatorname{deg} f_{0}=\operatorname{deg} f_{i}=0$.

Remarks. 1) Comparisson of the asymptotical expansions for the components in $H^{0}(\mathcal{V}, \mathbb{Q})$ and $H^{2}(\mathcal{V}, \mathbb{Q})$ of the functions $I$ and $J$ in orders $h^{0}$ and $h^{-1}$ uniquely determines the change of variables that transforms $I$ to $J$ (see Section 7).

2) The assumption $c\left(\mathcal{T}_{Y}\right) \geq 0$ guarantees that $\operatorname{deg} q^{d} \geq 0$ for all $d \in$ $\Lambda-0$. If $c\left(\mathcal{T}_{Y}\right)$ is positive, then the change of coordinates transforming $I$ to $J$ reduces to the multiplication by $\exp (h(q) / \hbar)$ where $h(q)$ is a polynomial supported at $\left\{d \in \Lambda \mid \int_{[d]} c\left(\mathcal{T}_{Y}\right)=1\right\}$. Thus the functions $I$ and $J$ coincide whenever this support is empty (for instance, if $c\left(\mathcal{T}_{Y}\right)$ is a multiple of a positive integer class).

3) In the opposite case $\sum v_{a}=\sum u_{j}$ the manifold $Y$ is a Calabi-Yau toric complete intersection (in the broad sense that $c\left(\mathcal{T}_{Y}\right)=0$ ). Then $\operatorname{deg} q_{i}=0$ for all $i, h(q)=0$ and

$$
\exp f_{0}=\sum_{d: D_{j}(d) \geq 0} q^{d} \frac{L_{1}(d) ! \ldots L_{l}(d) !}{D_{1}(d) ! \ldots D_{N}(d) !}
$$

This and other components of the vector-function $I$ are generalized hypergeometric functions. 
Example: toric manifolds themselves. Taking $l=0$ in Theorem 0.1 we arrive at the case $Y=X$ of Gromov - Witten theory on non-singular toric symplectic manifolds with $c\left(\mathcal{T}_{X}\right) \geq 0$. We describe further simplifications which occur in this case.

(a) If the anti-canonical class of the toric manifold is positive then $I=J_{X}$. The proof consists in elementary verification of the asymptotocal expansion $I=\exp \left(\left(t_{0}+p \log q\right) / \hbar\right)(1+o(1 / \hbar))$. In particular, symbols of differential operators annihilating $I$ yield the relations

$$
u_{1}^{D_{1}(d)} \ldots u_{N}^{D_{N}(d)}=q^{d}
$$

in the quantum cohomology algebra of $X$ (see Corollary 0.4 below). This result agrees with the multiplicative structure in Floer cohomology of the loop space $L X$ constructed in [7] by the method of generating functions (and based on discretization of loops) and with the results in [3, 16] on quantum cohomology of positive toric manifolds.

(b) If the anti-canonical class of the toric manifold is semi-positive then

$$
I=e^{\left(t_{0}+p \log q\right) / \hbar}\left[1+p_{1} f_{1}(q) / \hbar+\ldots+p_{k} f_{k}(q) / \hbar+o(1 / \hbar)\right]
$$

and differs from $J_{X}$ only by the change of variables $\log q_{i} \mapsto \log q_{i}+f_{i}(q)$. We illustrate this case by the following instructive example which also exhibits symplectic invariance of Gromov - Witten theory.

Let $X_{1}$ and $X_{2}$ be the toric 3 -folds obtained by projectivization of the following 3-dimensional vector bundles over $\mathbb{C} P^{1}: \mathcal{O}(-1) \oplus \mathcal{O}(-1) \oplus \mathcal{O}$ for $X_{1}$ and $\mathcal{O}(-2) \oplus \mathcal{O} \oplus \mathcal{O}$ for $X_{2}$. The bundles are topologically (and symplecticly) equivalent and the manifolds $X_{1}$ and $X_{2}$ are symplectomorphic. They are not isomorphic however as complex manifolds, and we shell see how the same Gromov - Witten invariants emerge from different computational procedures.

The manifolds $X_{1}, X_{2}$ are obtained by factorization of $\mathbb{C}^{5}$ by the torus $T_{\mathbb{C}}^{2}$ embedded into the 5 -torus of diagonal matrices as prescribed by the matrices $M_{1}^{t}$ and $M_{2}^{t}$ respectively:

$$
M_{1}=\left[\begin{array}{rrrrr}
1 & 1 & 0 & -1 & -1 \\
0 & 0 & 1 & 1 & 1
\end{array}\right], M_{2}=\left[\begin{array}{rrrrr}
1 & 1 & 0 & 0 & -2 \\
0 & 0 & 1 & 1 & 1
\end{array}\right]
$$

The columns of the matrix $M$ represent the classes $u_{1}, \ldots, u_{5}$ as linear combinations of a basis $p_{1}, p_{2}$ in $H^{2}(X)$ : for $X_{1}$ we have $u_{1}=u_{2}=p_{1}, u_{3}=p_{2}, u_{4}=$ 
$u_{5}=p_{2}-p_{1}$. Using upper-case notations for $X_{2}$ we get $U_{1}=U_{2}=p_{1}, U_{3}=$ $U_{4}=p_{2}, U_{5}=p_{2}-2 p_{1}$. We refer the reader to Section 3 for a detailed combinatorial description of cohomological invariants of toric manifolds. Using that description we find the multiplicative relations $u_{1} u_{2}=0, u_{3} u_{4} u_{5}=0$ and $U_{1} U_{2}=0, U_{3} U_{4} U_{5}=0$ in the cohomology algebra and observe that they are effectively the same:

$$
H^{*}(X)=\mathbb{Z}\left[p_{1}, p_{2}\right] /\left(p_{1}^{2}, p_{2}^{3}-2 p_{2}^{2} p_{1}\right) .
$$

The Kähler cone in both cases consists of $p_{1} t_{1}+p_{2} t_{2}$ with $t_{1}, t_{2}>0$. The anti-canonical class $\sum u_{j}=\sum U_{j}=3 p_{2}$, and the semigroup algebra $\mathbb{Q}[[\Lambda]]$ of identifies with $\mathbb{Q}\left[\left[q_{1}, q_{2}\right]\right]$ with the grading $\operatorname{deg} q_{1}=0, \operatorname{deg} q_{2}=3$.

The series $I$ corresponding to $X_{1}$ has the form

$$
\begin{gathered}
I_{1}=e^{\left(t_{0}+p_{1} \log q_{1}+p_{2} \log q_{2}\right) / \hbar} \times \\
\sum_{d_{1}, d_{2}=0}^{\infty} \frac{q_{1}^{d_{1}} q_{2}^{d_{2}} \prod_{m=-\infty}^{0}\left(p_{2}-p_{1}+m \hbar\right)^{2}}{\prod_{m=1}^{d_{1}}\left(p_{1}+m \hbar\right)^{2} \prod_{m=1}^{d_{2}}\left(p_{2}+m \hbar\right) \prod_{m=-\infty}^{d_{2}-d_{1}}\left(p_{2}-p_{1}+m \hbar\right)^{2}}
\end{gathered}
$$

It has the asymptotics $\exp \left(\left(t_{0}+p \log q\right) / \hbar\right)[1+o(1 / \hbar)]$ and thus coincides with $J_{X}$.

The series $I_{1}$ is annihilated by the differential operators

$$
\begin{aligned}
\Delta_{1} & =\left(\hbar \frac{\partial}{\partial \log q_{1}}\right)^{2}-q_{1}\left(\hbar \frac{\partial}{\partial \log q_{2}}-\hbar \frac{\partial}{\partial \log q_{1}}\right)^{2}, \\
\Delta_{2} & =\hbar \frac{\partial}{\partial \log q_{2}}\left(\hbar \frac{\partial}{\partial \log q_{2}}-\hbar \frac{\partial}{\partial \log q_{1}}\right)^{2}-q_{2} .
\end{aligned}
$$

Thus the rlations

$$
p_{1}^{2}=q_{1}\left(p_{2}-p_{1}\right)^{2}, p_{2}\left(p_{2}-p_{1}\right)^{2}=q_{2}
$$

describe the quantum deformation of the cohomology algebra of $X$.

The series $I$ corresponding to $X_{2}$ has the form (we use the upper-case notations $Q_{1}, Q_{2}$ instead of $\left.q_{1}, q_{2}\right)$ :

$$
\begin{gathered}
I_{2}=e^{\left(t_{0}+p_{1} \log Q_{1}+p_{2} \log Q_{2}\right) / \hbar} \times \\
\sum_{d_{1}, d_{2}=0}^{\infty} \frac{Q_{1}^{d_{1}} Q_{2}^{d_{2}} \Pi_{m=-\infty}^{0}\left(p_{2}-2 p_{1}+m \hbar\right)}{\prod_{m=1}^{d_{1}}\left(p_{1}+m \hbar\right)^{2} \Pi_{m=1}^{d_{2}}\left(p_{2}+m \hbar\right)^{2} \Pi_{m=-\infty}^{d_{2}-2 d_{1}}\left(p_{2}-2 p_{1}+m \hbar\right)} .
\end{gathered}
$$


It has the asymptotics $\exp \left(\left(t_{0}+p \log Q\right) / \hbar\right)\left[1+\left(2 p_{1}-p_{2}\right) f\left(Q_{1}\right) / \hbar+o(1 / \hbar)\right]$ where

$$
f\left(Q_{1}\right)=\sum_{d_{1}=1}^{\infty} \frac{\left(2 d_{1}-1\right) !}{\left(d_{1} !\right)^{2}} Q_{1}^{d_{1}} .
$$

We have to put therefore $q_{1}=Q_{1} \exp \left(2 f\left(Q_{1}\right)\right), q_{2}=Q_{2} \exp \left(-f\left(Q_{1}\right)\right)$.

In fact the inverse change of variables is given by the simple formulas

$$
\begin{gathered}
Q_{1}=\frac{q_{1}}{\left(1+q_{1}\right)^{2}}, Q_{2}=q_{2}\left(1+q_{1}\right), \\
Q_{2} \frac{\partial}{\partial Q_{2}}=q_{2} \frac{\partial}{\partial q_{2}}, Q_{2} \frac{\partial}{\partial Q_{2}}-2 Q_{1} \frac{\partial}{\partial Q_{1}}=\frac{1+q_{1}}{1-q_{1}}\left(q_{2} \frac{\partial}{\partial q_{2}}-2 q_{1} \frac{\partial}{\partial q_{1}}\right) .
\end{gathered}
$$

With these formulas at hands it is straightforward to check that the change of variables transforms the system of differential equations

$$
\begin{gathered}
\hbar^{3}\left(Q_{2} \frac{\partial}{\partial Q_{2}}\right)^{2}\left(Q_{2} \frac{\partial}{\partial Q_{2}}-2 Q_{1} \frac{\partial}{\partial Q_{1}}\right) I=Q_{2} I \\
\left(Q_{1} \frac{\partial}{\partial Q_{1}}\right)^{2} I=Q_{1}\left(Q_{2} \frac{\partial}{\partial Q_{2}}-2 Q_{1} \frac{\partial}{\partial Q_{1}}\right)\left(Q_{2} \frac{\partial}{\partial Q_{2}}-2 Q_{1} \frac{\partial}{\partial Q_{1}}-1\right) I
\end{gathered}
$$

satisfied by $I=I_{2}$ to the system $\Delta_{1} I=0, \Delta_{2} I=0$ satisfied by $I=I_{1}$. This guarantees that $I_{2}(Q(q))=I_{1}(q)$ and finally gives rise to the same description of the quantum cohomology algebra.

Our proof of Theorem 0.1 is based on an equivariant generalization of the Gromov - Witten theory.

Let $\mathcal{V}$ be a holomorphic $l$ - dimensional vector bundle over the $n$ - dimensional Kähler manifold $X$ equivariant with repect to a hamiltonian Killing action of a torus $G$. It was explained in 10 how to extend the Gromov Witten theory to the $G$ - super - manifolds $(X, \mathcal{V})$ of dimension $(n, l)$ in the case of convex $\mathcal{V}$ (i.e. bundles with all fibers spanned by global holomorphic sections). 2

The equivariant quantum cohomology algebra of the super - manifold $(X, \mathcal{V})$ is a deformation of the cup - product in the equivariant cohomology algebra $H_{G}^{*}(X, \mathbb{Q})$ provided with the Poincare pairing $\langle f, g\rangle=\int_{[X]} f g \operatorname{Euler}(\mathcal{V})$

\footnotetext{
${ }^{2}$ The idea of the construction is due to M. Kontsevich (see [12, 10]) while the terminology of super-manifolds in this context was introduced by A. Schwarz [17].
} 
with values in $H_{G}^{*}($ point $)=H^{*}(B G, \mathbb{Q})$. Here the equivariant top Chern class $\operatorname{Euler}(\mathcal{V})$ is assumed to be invertible over the field of fractions of the polynomial algebra $H^{*}(B G, \mathbb{Q}) \simeq \mathbb{Q}\left[\lambda_{1}, \ldots, \lambda_{\operatorname{dim} G}\right]$. Respectively, the equivariant quantum cohomology algebra and the quantum cohomology $\mathcal{D}$-module of the super-manifold are defined over this field of fractions. It is important however that the structural constants $\langle a \circ b, c\rangle$ and the components $\left\langle J_{\mathcal{V}}, a\right\rangle$ of the corresponding solution vector-function are defined over the polynomial algebra $\mathbb{C}[\lambda]$ (as some equivariant Poincare pairings with the top Chern classes of suitable vector bundles over the moduli spaces of stable maps to $X$, see Section 1). In the non-equivariant limit to $\lambda=0$ the algebra $\left(H_{G}^{*}(X) / \operatorname{ker}\langle\cdot, \cdot\rangle\right)$ degenerates to $H^{*}(\mathcal{V})$, and the corresponding structural constants and solutions turn into their counterparts in the Gromov-Witten theory on the complete intersection $Y \subset X$ defined by a generic holomorphic section of $\mathcal{V}$. This allows to obtain Theorem 0.1 as the specialization to $\lambda=0$ of the following result about toric super-manifolds.

Let us consider the equivariant cohomology algebra $H_{T^{N}}^{*}(X)$ of the toric manifold $X=\mathbb{C}^{N} / / T^{k}$ provided with the action of the torus $T^{N}$ of diagonal unitary matrices. The coefficient algebra $H^{*}\left(B T^{N}\right)$ of the equivariant theory is canonically isomorphic to the algebra $\mathbb{Z}\left[\lambda_{1}, \ldots, \lambda_{N}\right]$ of polynomial functions on $\operatorname{LieT}^{N}$. The algebra $H_{T^{N}}^{*}(X)$ is multiplicatively generated over $\mathbb{Z}[\lambda]$ by the equivariant counterpart $p_{1}, \ldots, p_{k} \in H^{2}(X)$ of a basis in $H^{2}(X)$. The equivariant classes $u_{1}, \ldots, u_{N} \in H_{G}^{2}(X)$ Poincare-dual to the $G$-invariant toric hypersurfaces corresponding to the coordinate hyperplanes in $\mathbb{C}^{N}$ are some linear combinations

$$
u_{j}=\sum_{i=1}^{k} p_{i} m_{i j}-\lambda_{j}, j=1, \ldots, N,
$$

of these generators. The relations between the generators $p_{i}$ can be written in the form (see Section 3):

$$
u_{j_{1}} \ldots u_{j_{r}}=0, j_{1}<\ldots<j_{r}
$$

whenever the toric hypersurfaces corresponding to $u_{j_{1}}, \ldots, u_{j_{r}}$ have empty intersection.

Let $\mathcal{V}$ denote the direct sum of $l \geq 0$ non-negative $T^{N}$-equivariant line 
bundles over $X$ with the non-zero equivariant 1-st Chern classes

$$
v_{a}=\sum_{j=1}^{N} p_{i} l_{i a}-\lambda_{a}^{\prime}, a=1, \ldots, l .
$$

The variables $\left(\lambda^{\prime}, \ldots, \lambda_{l}^{\prime}\right)$ here stand for the generators of $H^{*}\left(B T^{l}\right)$ where the torus $T^{l}$ acts fiberwise on the bundle $\mathcal{V}$. (One may think of the toric supermanifold $(X, \mathcal{V})$ as of the symplectic reduction of the super-space $\left(\mathbb{C}^{N}, \mathbb{C}^{l}\right)$ by the torus $T^{k}$ embedded into the maximal torus $G=T^{N} \times T^{l}$ by means of the matrix $\left(m_{i j} \mid l_{i a}\right)$.)

Denote $J_{\mathcal{V}}\left(t_{0}, \log q, \hbar^{-1}\right)$ the solution formal vector-function of the equivariant Gromov - Witten theory on the super-manifold $(X, \mathcal{V})$. Define another formal vector-function $I_{\mathcal{V}}\left(t_{0}, \log q, \hbar^{-1}\right)$ by the formal series $(*)$. Coefficients of both formal functions are $G$-equivariant cohomology classes of $X$ over $\mathbb{Q}\left[\lambda, \lambda^{\prime}\right]$. In the limit $\left(\lambda, \lambda^{\prime}\right)=0$ the functions $J_{\mathcal{V}}$ and $I_{\mathcal{V}}$ yield $J$ and $I$ respectively.

Theorem 0.2. Suppose that 1-st Chern class $\sum u_{j}-\sum v_{a}$ of the supermanifold $(X, \mathcal{V})$ is non-negative. Then $J_{\mathcal{V}}$ coincides with $I_{\mathcal{V}}$ up to a weightedhomogeneous triangular change of variables:

$$
t_{0} \mapsto t_{0}+f_{0}(q) \hbar+\sum \lambda_{j} g_{j}(q)+h(q), \log q_{i} \mapsto \log q_{i}+f_{i}(q), i=1, \ldots, k
$$

where $f_{0}, f_{i}, g_{j}, h$ are weighted-homogeneous formal $q$-series with $\operatorname{deg} f_{0}=$ $\operatorname{deg} f_{i}=\operatorname{deg} g_{j}=0, \operatorname{deg} h=1$ supported at $\Lambda-0$.

Corollary 0.3. Suppose that a linear differential operator $D(\hbar \partial / \partial \log q, q, \lambda, \hbar)$ with coefficients in $\mathbb{C}[\lambda, \hbar][[\Lambda]]$ annihilates the vectorfunction $I_{\mathcal{V}}$ transformed to the new variables. Then the relation $D(p \circ, q, \lambda, 0)$ $=0$ holds in the quantum cohomology algebra of the super-manifold $(X, \mathcal{V})$. In particular, in the quantum cohomology algebra of the complete intersection $Y \subset X($ with $\operatorname{dim} Y>1)$ we have $\langle a, D(p \circ, q, 0,0) \circ b\rangle=0$ for any $a, b \in$ $H^{*}(\mathcal{V}) \subset H^{*}(Y)$.

Remarks. 4) The change of variables transforming $I_{\mathcal{V}}$ to $J_{\mathcal{V}}$ is uniquely determined by the asymptotics of $I_{\mathcal{V}}$ modulo $\hbar^{-2}$.

5) The $q^{d}$-term in $\left\langle a, b \circ p_{i_{1}} \circ \ldots \circ p_{i_{r}}\right\rangle$ has the following enumerative meaning: it is the (virtual) number of degree $d$ holomorphic maps $\mathbb{C} P^{1} \rightarrow Y$ which send a given generic configuration of $r+2$ distinct points in $\mathbb{C} P^{1}$ to the 
given generic cycles $a, b$ and $r$ given generic hypersurfaces Poincare-dual to $p_{i_{1}}, \ldots, p_{i_{r}}$ respectively.

6) Due to a somewhat subtle relationship between the quantum cohomology algebras of $Y$ and $(X, \mathcal{V})$ it seems to be dangerous to deduce enumerative corollaries about $Y$ directly from differential equations for $J$ (instead of $J_{\mathcal{V}}$ or $\left.J_{Y}\right)$. We do not know counter-examples however. In many cases such corollaries can be justified by means of additional dimensional or Hodge-theoretic arguments. In particular, if $\oplus H^{r, r}(Y) \subset H^{*}(\mathcal{V}, \mathbb{C})$ (for example if $Y \subset X$ is a hypersurface of odd dimension), we have $\left\langle a, J_{Y}\right\rangle=\langle a, J\rangle$ for all $a \in H^{*}(Y)$. In this case $D(p \circ, q, 0)=0$ if the differential operator $D(\hbar \partial / \partial \log q, q, \hbar)$ annihilates $I$ transformed to the new variables as described in Theorem 0.1.

Consider now the differential equations satisfied by $I_{\mathcal{V}}$. Put

$$
\begin{gathered}
\partial_{j}=\sum_{i} m_{i j} \hbar \partial / \partial \log q_{i}-\lambda_{j}, j=1, \ldots, N, \\
\partial_{a}^{\prime}=\sum_{i} l_{i a} \partial / \partial \log q_{i}-\lambda_{a}^{\prime}, a=1, \ldots, l .
\end{gathered}
$$

For each $d \in \Lambda$ with $D_{1}(d), \ldots, D_{N}(d) \geq 0$ we introduce the differential operator (see [9]):

$$
\Delta_{d}=\Pi_{j} \Pi_{m=0}^{D_{j}(d)-1}\left(\partial_{j}-m \hbar\right)-q^{d} \Pi_{a} \Pi_{m=1}^{L_{a}(d)}\left(\partial_{a}^{\prime}+m \hbar\right) .
$$

It follows easily from the detailed description of the equivariant cohomology algebra $H_{G}^{*}(X)$ given in Section 3 that $\Delta_{d} I_{\mathcal{V}}=0$. (In fact $I_{\mathcal{V}}$ may satisfy some stronger differential equations.)

Denote $\hat{\Delta}_{d}$ the polynomial

$$
u_{1}^{D_{1}(d)} \ldots u_{N}^{D_{N}(d)}-q^{d} v_{1}^{L_{1}(d)} \ldots v_{l}^{L_{l}(d)}
$$

in the quantum cohomology algebra of the non-negative toric complete intersection $Y \subset X$.

Corollary 0.4. Suppose that for the toric complete intersection $Y \subset X$ we have $J_{\mathcal{V}}=I_{\mathcal{V}}$ (for instance due to the causes described in Remark 2). Then $\left\langle a, b \circ \hat{\Delta}_{d}\right\rangle=0$ for any $a, b \in H^{*}(\mathcal{V}) \subset H^{*}(Y)$.

We would like to emphasise the hypergeometric character of the function $I_{\mathcal{V}}$. It is easy to see that the differential operators $\Delta_{d}$ annihilate also the 
following hypergeometric integrals:

$$
\begin{gathered}
\int_{\Gamma \subset E_{q}} e^{\left(\sum u_{j}-\sum v_{a}\right) / \hbar} u_{1}^{\lambda_{1} / \hbar} \ldots u_{N}^{\lambda_{N} / \hbar} v_{1}^{\lambda_{1}^{\prime} / \hbar} \ldots v_{l}^{\lambda_{l}^{\prime} / \hbar} \times \\
\times \frac{d \log u_{1} \wedge \ldots \wedge d \log u_{N} \wedge d v_{1} \wedge \ldots \wedge d v_{l}}{d \log q_{1} \wedge \ldots \wedge d \log q_{k}} .
\end{gathered}
$$

Here $\Gamma_{q}$ is a suitable real $N-k+l$-dimensional (non-compact) cycle in the complex $N-k+l$-dimensional variety

$$
E_{q}=\left\{(u, v) \mid \Pi_{j=1}^{N} u_{j}^{m_{i j}}=q_{i} \Pi_{a=1}^{l} v_{a}^{l_{i a}}, i=1, \ldots, k\right\}
$$

provided with the local coefficient system $u^{\lambda / \hbar} v^{\lambda^{\prime} / \hbar}$.

Example: a mirror theorem. Partition the variables $u_{1}, \ldots, u_{N}$ into $l+1$ groups and denote $F_{a}$ the sum of $u_{j}$ in each group, $a=0, \ldots, l$. Define the matrix $\left(l_{i a}\right)$ in such a way that $v_{a}=F_{a}(u), a=1, \ldots, l$, correspond to convex line bundles on $X$. In the limit $\lambda^{\prime}=0, \lambda=0$ the above integral evaluated explicitly over $\mathbb{R}_{+}^{l}$ in $d v$ reduces to

$$
\int_{\Gamma_{q}^{\prime} \subset X_{q}^{\prime}} e^{F_{0}(u) / \hbar} \frac{d \log u_{1} \wedge \ldots \wedge d \log u_{N}}{\left(1-F_{1}(u)\right) \ldots\left(1-F_{l}(u)\right) d \log q_{1} \wedge \ldots \wedge d \log q_{k}}
$$

where $X_{q}^{\prime}=\left\{u \mid \Pi_{j} u_{j}^{m_{i j}}=q_{i}, i=1, \ldots, k\right\}$. Further reduction by Cauchy residue formula yields

$$
\text { (**) } \int_{\gamma_{q} \subset Y_{q}^{\prime}} e^{F_{0}(u) / \hbar} \frac{d \log u_{1} \wedge \ldots \wedge d \log u_{N}}{d F_{1} \wedge \ldots \wedge d F_{l} \wedge d \log q_{1} \wedge \ldots \wedge d \log q_{k}} .
$$

Here $\gamma_{q}$ can be understood as Morse-theoretic cycles of the function $\operatorname{Re} F_{0}$ restricted to the $N-k-l$-dimensional manifold

$$
Y_{q}^{\prime}=\left\{u \mid \Pi_{j} u_{j}^{m_{i j}}=q_{i}, i=1, \ldots, l, F_{a}(u)=1, a=1, \ldots, l\right\} .
$$

It is not hard to see that all components of the vector-function $I$ are described by such integrals with suitable $\gamma_{q}$. P This constitutes the content of the mirror symmetry between the toric complete intersections $Y$ and $Y^{\prime}$.

\footnotetext{
${ }^{3}$ In general the converse is not true - different toric manifolds $X=\mathbb{C}^{N} / / T^{k}$ obtained by the reduction on different generic levels of the momentum map have different cohomology algebras $H^{*}(\mathcal{V})$ and may give rise to different $I$ whose integral representations differ by the choice of cycles only.
} 
Indeed:

(a) In the case of a Calabi-Yau toric complete intersection $Y$ we have $\sum u_{j}=\sum v_{a}$ and thus $F_{0}(u)=0$. The affine varieties $X_{q}^{\prime}$ in this case can be compactified (see [2]) to the toric varietiy $\hat{X}^{\prime}$ with the momentum polyhedron polar to that for $X$. The varieties $Y_{q}^{\prime}$ compactify to (singular) Calabi-Yau complete intersections $\hat{Y}_{q}^{\prime} \subset \hat{X}^{\prime}$. The forms $d \log u / d v \wedge d \log q$ extend to holomorphic volume forms $\omega_{q}$ on desingularizations of $\hat{Y}_{q}^{\prime}$. Thus the components of the hypergeometric series $I$ identify with the periods $\int_{\gamma_{\log q}} \omega_{q}$ responsible for variations of complex structures in a family Calabi-Yau manifolds birationally isomorphic to $\hat{Y}_{q}^{\prime}$.

According to V.Batyrev [2] the $q$-family of Calabi-Yau manifolds is mirror - symmetric to the original family of toric complete intersections $Y$, and thus our Theorem 0.1 confirms the mirror conjecture for Calabi - Yau toric complete intersections formulated in detail in [凹].

(b) In the lecture [9] we suggested a generalization of the mirror conjecture beyond the class of Calabi - Yau manifolds. f In this generalization the quantum cohomology $\mathcal{D}$-module of a compact Kähler manifold $Y$ should be equivalent, up to some change of variables, to the $\mathcal{D}$-module generated by the oscillating integrals $\int e^{f_{q} / \hbar} \omega_{q}$ defined by a suitable family $\left(Y_{q}^{\prime}, \omega_{q}, f_{q}\right)$ of (possibly non-compact) complex algebraic manifolds $Y_{q}^{\prime}$ (of the same dimension as $Y$ ) provided with holomorphic volume forms $\omega_{q}$ and holomorphic phase functions $f_{q}$. This generalisation was confirmed for toric Fano manifolds in [9], for Fano and Calabi-Yau projective complete intersections in [10] and for flag manifolds of the series $A$ in 11$]$. Thus Theorem 0.1 along with the integral representation $(* *)$ proves our generalized mirror conjecture for non-negative toric complete intersections described at the beginning of this example.

The remaining part of the paper contains a proof of Theorem 0.2. It exploits some general properties of equivariant Gromov - Witten invariants of super-manifolds $(X, \mathcal{V})$ described in [10] in the setting of convex Kähler manifolds $X$. Foundations of the Gromov - Witten theory for general $X$ were developed recently by several groups of authors (see for instance [14]). It appears that these results admit staighforward equivariant generalizations.

\footnotetext{
${ }^{4} \mathrm{~A}$ similar generalization was recently proposed in [6].
} 
However there is no ready reference for such a generalization in the literature. In Section 1 we describe the properties of the equivariant Gromov-Witten theory we use in this paper in the axiomatic form. These properties have been verified in 10 for convex $X$. Thus the proof of Theorems 0.1 and 0.2 given in this paper is complete in the case of non-negative complete intersections in products of projective spaces (such products are convex), and in the case of more general toric manifolds still should be complemented by a verification of the axioms.

1. Moduli spaces of stable maps. Let $(C, x)$ be a compact connected complex algebraic curve with at most double singular points and with $r$ pairwise distinct non-singular marked points $x=\left(x_{1}, \ldots, x_{r}\right)$. We will assume that $C$ has the arithmetic genus $\operatorname{dim} H^{1}(C, \mathcal{O})=0$. Two holomorphic maps $(C, x) \rightarrow M,\left(C^{\prime}, x^{\prime}\right) \rightarrow M$ of two such curves to a complex manifold $Y$ are called equivalent if they are identified by a holomorphic isomorphism $(C, x) \rightarrow\left(C^{\prime}, x^{\prime}\right)$. A holomorphic map $(C, x) \rightarrow M$ is called stable (see [12]) if it does not admit non-trivial infinitesimal automorphisms. A stable map may have a non-trivial finite group of discrete automorphisms.

The degree of a holomorphic map $(C, x) \rightarrow M$ is defined as the homology class $d \in H_{2}(M)$ it represents.

Denote $M_{r, d}$ the set of equivalence classes of degree $d$ genus 0 stable maps $(C, x) \rightarrow M$ with $r$ marked points. For a compact projective variety $M$ the set $M_{r, d}$ has a natural structure of a compact complex algebraic variety (see [12, 5]). We will call the spaces $M_{r, d}$ the moduli spaces of stable maps.

If $M$ is a homogeneous Kähler space of a compact semi-simple Lie group, the moduli spaces $M_{n, d}$ are known [5] to be non-singular orbifolds i.e. local quotients of non-singular spaces by finite groups. In particular the spaces have natural fundamental cycles providing Poincare duality over $\mathbb{Q}$. For general $M$ it is still convenient to think of the moduli spaces $M_{n, d}$ as of singular orbifolds i.e. local quotients of singular spaces by finite groups. The finite groups in question are the discrete automorphism groups of stable maps, and many local constructions in the moduli spaces become transparent only after passing to their local "unquotient" coverings. Vector bundles and their characteristic classes provide important examples of the orbifold ideology. By a vector bundle over $M_{n, d}$ we mean a sheaf which is locally identified with the sheaf of invariant sections of a natural local vector bundle on such coverings. The structural group $G$ of the bundle acts with at most finite stabilizers 
on the total space of corresponding principal bundle $P \rightarrow M_{n, d}$. In this case the rational coefficient equivariant cohomology algebra $H_{G}^{*}(P, \mathbb{Q})$ naturally identifies with $H^{*}\left(M_{n, d}, \mathbb{Q}\right)$. Then the augmentation homomorphism $H^{*}(B G, \mathbb{Q}) \rightarrow H_{G}^{*}(P, \mathbb{Q})$ induced by the $G$-equivariant map $P \rightarrow p t$ defines characteristic classes of the bundle. Talking about vector bundles over the moduli spaces, their Euler and Chern classes we will always have this orbifold subtlety in mind.

According to [15, 14] the moduli spaces $M_{n, d}$ can be provided with virtual fundamental cycles — rational homology classes of $M_{r, d}$ satisfying the axioms [13] of Gromov - Witten theory. We call the virtual fundamental cycles Gromov - Witten classes. Both the axioms and their realization by means of Gromov - Witten classes allow dependence of $M$ on parameters.

Suppose that a compact Lie group $G$ acts on a compact Kähler manifold $M$ by hamiltonian automorphisms of the Kähler structure. Then $G$ acts naturally on the Moduli spaces $M_{r, d}$. Let $B \subset B G$ be a finite-dimensional approximation to the classifying space $B G$ of principal $G$-bundles, and $M_{B} \rightarrow B$

- the associated $M$-bundle. The bundle can be considered as a family of compact Kähler manifolds. This allows to construct parametric Gromov Witten classes in the moduli spaces $\left(M_{r, d}\right)_{B} \rightarrow B$. Exhausting $B G$ by the finite-dimensional approximations one can construct $G$-equivariant GromovWitten classes $\left[M_{r, d}\right]$. The recent progress [15, 14] in foundations of Gromov - Witten theory leaves little doubt that the "virtual fundamental class" approach is consistent with the axioms [13, 10] of equivariant Gromov - Witten theory.

We describe below a variant of the axioms in the form convenient for applications in the present paper.

(1) The equivariant Gromov - Witten class defines an $H^{*}(B G, \mathbb{Q})$ - linear function $\int_{\left[M_{r, d}\right]}: H_{G}^{*}\left(M_{r, d}, \mathbb{Q}\right) \rightarrow H^{*}(B G, \mathbb{Q})$ of homogeneity degree $-2\left[\left(c_{1}\left(\mathcal{T}_{M}\right), d\right)+\operatorname{dim}_{\mathbb{C}} M+r-3\right]$. The moduli spaces $M_{r, 0}$ are isomorphic to $M \times \overline{\mathfrak{M}}_{r}$ where $\overline{\mathfrak{M}}_{r}$ is the Deligne - Mumford compactification of the moduli space $\mathfrak{M}_{r}$ of ordered $r$-tuples of distinct points on $\mathbb{C} P^{1}$. The Gromov - Witten classes $\left[M_{r, 0}\right]$ are (equivariant) fundamental cycles of the manifolds $M_{r, 0}$ for $r \geq 3$ and are not defined for $r=0,1,2$.

Remark. It is crucial for applications in this paper that the equivariant Gromov - Witten class is indeed defined over the polynomial algebra $H^{*}(B G, \mathbb{Q})$ and not over its field of fractions. 
(2) There exist natural equivariant forgetting maps fgt $_{s}: M_{r+1, d} \rightarrow M_{r, d}$ defined (see [12, 5]) by forgetting the marked point $x_{s}, 1 \leq s \leq r+1$. The fiber of fgt $_{s}$ over the point represented by a stable map $(C, x) \rightarrow M$ is canonically isomorphic to the quotient of $C$ by the finite group of automorphisms of the stable map.

(3) There exist natural equivariant evaluation maps $\mathrm{ev}_{s}: M_{r, d} \rightarrow M$ defined by evaluation of a stable map at the marked point $x_{s}$. The 3-point correlators

$$
\langle a, b, c\rangle:=\sum_{d} q^{d} \int_{\left[M_{3, d}\right]} \operatorname{ev}_{1}^{*}(a) \operatorname{ev}_{2}^{*}(b) \operatorname{ev}_{3}^{*}(c)
$$

are structural constants $\langle a \circ b, c\rangle$ of a (super) commutative associative Frobenius algebra structure on $\left(H_{G}^{*}(M),\langle\cdot, \cdot\rangle\right)$ with the unity 1 . Here $q^{d}$ stands for the element in the group ring of the lattice $H_{2}(M)$ corresponding to $d \in H_{2}(M)$, and the (super) symmetric bilinear form $\langle a, b\rangle$ is the (equivariant) Poincare pairing $\int_{M} a b$. The axiom means that the new $H^{*}(B G, \mathbb{Q}[[q]])$ bilinear multiplication $\circ$ on $H_{G}^{*}(M, \mathbb{Q}[[q]])$ defined by

$$
\langle a \circ b, c\rangle:=\langle a, b, c\rangle \forall a, b, c \in H_{G}^{*}(M)
$$

is associative, and that the class $1 \in H_{G}^{0}(M)$ plays the role of the unity:

$$
\langle a, b, 1\rangle=\langle a, b\rangle \forall a, b \in H_{G}^{*}(M) .
$$

Here $\mathbb{Q}[[q]]$ is the formally completed semigroup algebra of the semigroup of degrees $d \in H_{2}(M)$ of compact holomorphic curves in $M$. The algebra $\left(H_{G}^{*}(M, \mathbb{Q}[[q]]), \circ\right)$ is called the (equivariant) quantum cohomology algebra of $M$. Reduced modulo the maximal ideal in $\mathbb{Q}[[q]]$, it identifies with the "classical" cohomology algebra $H_{G}^{*}(M, \mathbb{Q})$ since $M_{3,0}=M$.

(4) Let $d=\left(d_{1}, \ldots, d_{k}\right)$ denote coordinates of $d$ with respect to a basis in $H_{2}(M)$, and $p_{1}, \ldots, p_{k} \in H_{G}^{2}(M)$ represent the dual basis in $H^{2}(M) / H^{2}(B G)$. The $H^{*}(B G)$ - linear operators $p_{i}$ ○ of quantum multiplication by $p_{i}$ satisfy

$$
q_{i} \partial\left(p_{j} \circ\right) / \partial q_{i}=q_{j} \partial\left(p_{i} \circ\right) \partial q_{j} .
$$

These identities along with associativity and commutativity of the quantum multiplication mean that the following linear system of parial differential equations is consitent for any value of the parameter $\hbar \neq 0$ :

$$
\hbar \frac{\partial}{\partial t_{0}} s=s, \quad \hbar q_{i} \frac{\partial}{\partial q_{i}} s=p_{i} \circ s, \quad i=1, \ldots, k .
$$


(5) When $a$ runs an $H^{*}(B G)$-basis in $H_{G}^{*}(M)$, the following formal vector - functions $s_{a}$ run a basis of solutions to the above PDE system (see e. g. [10]). Define the (equivariant) class $s_{a}$ of $M$ (with appropriate coefficients) by

$$
\begin{gathered}
\forall b \in H_{G}^{*}(M)\left\langle s_{a}, b\right\rangle=\int_{M} e^{\left(t_{0}+p_{1} \log q_{1}+\ldots+p_{k} \log q_{k}\right) / \hbar} a b+ \\
\sum_{d \neq 0} q^{d} \int_{\left[M_{2, d}\right]} \frac{\mathrm{ev}_{1}^{*}\left(e^{\left(t_{0}+p_{1} \log q_{1}+\ldots+p_{k} \log q_{k}\right) / \hbar} a\right)}{\hbar-c} \operatorname{ev}_{2}^{*}(b) .
\end{gathered}
$$

Here $c$ is the (equivariant) 1-st Chern class of the following line bundle over $M_{2, d}$ called the universal cotangent line at the 1-st marked point. The forgetful map fgt f $_{3} M_{3, d} \rightarrow M_{2, d}$ (defined for $d \neq 0$ ) has the section $\mathrm{mk}_{1}: M_{2, d} \rightarrow M_{3, d}$ defined by marked point $x_{1}$ in each fiber. The universal cotangent line is defined as the conormal bundle to the hypersurface $\mathrm{mk}_{1}\left(M_{2, d}\right)$ in $M_{3, d}$. The fiber of this bundle at the point represented by the stable map $\left(C, x_{1}, x_{2}\right) \rightarrow M$ is the cotangent line $T_{x_{1}}^{*} C$.

In particular, this axiom implies (see [10]) that whenever a differential operator

$$
D\left(\hbar q_{1} \partial / \partial q_{1}, \ldots, \hbar q_{k} \partial / \partial q_{k}, q_{1}, \ldots, q_{k}, \hbar\right)
$$

with coefficients in $H^{*}(B G, \mathbb{C}[[q, \hbar]])$ annihilates simultaneously all functions $\left\langle s_{a}, 1\right\rangle, a \in H_{G}^{*}(M)$, the relation $D\left(p_{1} \circ, \ldots, p_{k} \circ, q_{1}, \ldots, q_{k}, 0\right)=0$ defined by the symbol of this operator holds true in the quantum cohomology algebra of $M$. The vector-function $J_{M}$ mentioned in the introduction is defined by $\forall a\left\langle s_{a}, 1\right\rangle=\left\langle a, J_{M}\right\rangle$.

(6) A holomorphic vector bundle $\mathcal{V}$ over $M$ is called convex if each fiber of $\mathcal{V}$ is generated by global holomorphic sections of $\mathcal{V}$. The Gromov Witten theory of the super-manifold $(M, \mathcal{V})$ is constructed by taking $f \mapsto$ $\int_{\left[M_{r, d}\right]} f \operatorname{Euler}\left(\mathcal{V}_{n, d}\right)$ on the role of Gromov - Witten classes. Here $\mathcal{V}_{n, d}$ is the vector bundle

$$
\mathcal{V}_{r, d}=\left(\operatorname{fgt}_{r+1}\right)_{*}\left(\operatorname{ev}_{r+1}^{*}(\mathcal{V})\right)
$$

over the moduli space $M_{n, d}$. The fiber of $\mathcal{V}_{r, d}$ over the point in $M_{r, d}$ represented by the degree $d$ stable map $\phi:(C, x) \rightarrow M$ is $H^{0}\left(C, \phi^{*}(\mathcal{V})\right)$ and has the complex dimension $\left(c_{1}(\mathcal{V}), d\right)$ since $H^{1}\left(C, \phi^{*}(\mathcal{V})\right)=0$ due to the convexity of the bundle. Evaluation at a marked point defines an epimorphism $\mathcal{V}_{n, d} \rightarrow \mathcal{V}$ of the bundles over $\mathrm{ev}_{s}: M_{n, d} \rightarrow M$. This allows to introduce the correlators $\langle a, b, c\rangle,\left\langle s_{a}, b\right\rangle$ for $a, b, c$ from the quotient $H^{*}(\mathcal{V})$ of $H^{*}(X, \mathbb{Q})$ 
by the kernel of the intersection form $\langle a, b\rangle=\int_{M} a b \operatorname{Euler}(\mathcal{V})$ and thus to define for the super-manifold $(X, \mathcal{V})$ the quantum cohomology algebra and the quantum cohomology $\mathcal{D}$-module satisfying the analogues of the axioms $(3),(4),(5)$ of the super-manifold $(X, \mathcal{V})$. Analogous axioms hold true in the equivariant setting provided that the convex bundle $\mathcal{V}$ is $G$-equivariant. Then Euler (.) means the equivariant Euler class. In particular, the vector-function $J_{\mathcal{V}}$ from Introduction is the counterpart of $J_{M}$ in the case of the (equivariant) super-manifolds $(X, \mathcal{V})$.

(7) Let $Y$ be a non-singular submanifold in $M$ defined as the zero locus of a section of the convex vector bundle $\mathcal{V}$. The inclusion $i: Y \subset M$ identifies the Frobenius algebra $\left(H^{*}(\mathcal{V}), a \mapsto \int_{M} a \operatorname{Euler}(\mathcal{V})\right)$ with the image of the homomorphism $i^{*}: H^{*}(M, \mathbb{Q}) \rightarrow H^{*}(Y, \mathbb{Q})$. Respectively, the inclusion of the moduli spaces $Y_{n, d} \rightarrow M_{n, i_{*} d}$ maps the Gromov - Witten class $\left[Y_{n, d}\right]$ to the Gromov - Witten class corresponding to the super-manifold $(X, \mathcal{V})$. This implies that the correlators $\langle a, b, c\rangle,\left\langle s_{a}, b\right\rangle$ for $Y$ between the classes $a, b, c$ induced from $M$ can be computed by integration over the Gromov - Witten classes $\left[M_{n, d}\right]$ against the Euler classes of $\mathcal{V}_{n, d}$. In particular the orthogonal projection $J$ of the vector-function $J_{Y}$ to $i^{*}\left(H^{*}(M, \mathbb{Q})\right)$ identifies with (the non-equivariant version of) $J_{\mathcal{V}}$.

(8) The Borel fixed point localization technique applies to the Gromov - Witten classes $\left[M_{r, d}\right]$. Consider the action of the torus $G$ on $M_{r, d}$. A fixed point of this action is represented by a map $\phi:(C, x) \rightarrow M$ such that each irreducible component of $C$ is mapped either to the fixed point manifold $M^{G}$ or onto a complex 1-dimensional orbit of $G_{\mathbb{C}}$. We will formulate the axiom in the special case where both the set of fixed points $M^{G}$ and the set of 1-dimensional orbits are finite. If it is the case, each connected component of the fixed point set in $M_{r, d}$ is (the quotient by a finite group of) a product $\Pi \overline{\mathfrak{M}}_{n}$ of Deligne-Mumford configuration spaces $\overline{\mathfrak{M}}_{n}$. Each factor in this product parametrizes configurations of marked and singular points on a 1-dimensional connected component of $\phi^{-1}\left(M^{G}\right) \subset C$. On each of the remaining irreducible components of $C$ the map $\phi$ covers a compactified 1dimensional orbit of $G$ (with certain multiplicity in which case it ramifies at the two compactifying fixed points). We will call these components edges of the curve $C$.

The localization of the equivariant Gromov - Witten class $\left[M_{r, d}\right]$ at the 
fixed point component in $M_{r, d}$ is

$$
f \mapsto \frac{1}{|A u t|} \int_{\Pi \overline{\mathfrak{M}}_{n}} \frac{f}{\text { Virtual Normal Euler Class }} .
$$

Here Aut is the automorphism group of a typical stable map $\phi$ from the fixed point component in question.

In order to describe the Virtual Normal Euler Class consider the vector spaces

$$
\mathcal{N}_{i}=H^{i}\left(C, \phi^{*}\left(\mathcal{T}_{M}\right)\right), i=0,1, \mathcal{N}_{2}=H^{0}\left(C, \mathcal{T}_{C}[x]\right), \mathcal{N}_{3}=\oplus_{y} T_{y}^{\prime} \otimes T_{y}^{\prime \prime},
$$

where $y$ runs double points of $C$ situated on the edges, and $T_{y}^{\prime}, T_{y}^{\prime \prime}$ are the tangent spaces to the two components of $C$ intersecting at $y$. Dimensions of the spaces $\mathcal{N}_{i}$ do not change along the connected fixed point component of $M_{r, d}$ and thus they form vector bundles over $\Pi \overline{\mathfrak{M}}_{n}$. These bundles carry natural infinitesimal actions of $G$. Indeed, this is obvoius for $\mathcal{N}_{0}$ and $\mathcal{N}_{1}$. The differential $\phi_{*}$ identifies the space $\mathcal{N}_{2}$ of infinitesimal automorphisms of $(C, x)$ with a $G$-invariant subspace in $\mathcal{N}_{0}$ due to stability of $\phi$. For a double point $y$ on the edge $C^{\prime}$ where $\phi$ is an $m$-fold covering map, the space $\left(T_{y} C\right)^{\otimes m}$ is identified with the tangent line to the closed 1-dimensional $G$-orbit at a fixed point and thus inherits an infinitesimal action of $G$. The Virtual Normal Euler Class is defined as the following combination of equivariant Euler classes of the bundles $\mathcal{N}_{i}$ :

$$
\frac{\operatorname{Euler}\left(\mathcal{N}_{0}\right) \operatorname{Euler}\left(\mathcal{N}_{3}\right)}{\operatorname{Euler}\left(\mathcal{N}_{1}\right) \operatorname{Euler}\left(\mathcal{N}_{2}\right)}
$$

Remark. One can use this formula in order to define the Gromov - Witten cycle in the equivariant theory. However the verification of the other axioms and especially - the polynomiality property (1) of the Gromov - Witten class to be defined over $H^{*}(B G, \mathbb{Q})=\mathbb{Q}[\lambda]$ - becomes then a nontrivial combinatorial task.

2. Graph spaces and universal line bundles. Let $X$ be a compact projective manifold. For $d \in H_{2}(X)$ we call the graph space and denote $G_{d}(X)$ the moduli space of genus 0 holomorphic maps to $\mathbb{C} P^{1} \times X$ of degree $(1, d)$. This space can be considered as a compactification of the space of degree $d$ holomorphic maps $\mathbb{C} P^{1} \rightarrow X$. The automorphism group of $\mathbb{C} P^{1}$ acts naturally on $G_{d}(X)$. 
Consider first the case $X=\mathbb{C} P^{N-1}=\left(\mathbb{C}^{N}-0\right) /(\mathbb{C}-0)$. Denote $L_{d}, d \in$ $\mathbb{Z}_{+}$, the projectivization $\operatorname{Proj}\left(\mathbb{C}^{N} \otimes S^{d}\left(\mathbb{C}^{2}\right)\right)$ of the space of degree $d$ vector binary forms $\left(P_{1}(\zeta: \xi): \ldots: P_{N}(\zeta: \xi)\right)$. It is a projective space of dimension $N d+N-1$ and carries a natural action of $\operatorname{Aut}\left(\mathbb{C} P^{1}\right)=P S L_{2}(\mathbb{C})$ too.

We define the $\operatorname{Aut}\left(\mathbb{C} P^{1}\right)$-equivariant map $\mu: G_{d}\left(\mathbb{C} P^{N-1}\right) \rightarrow L_{d}$. Consider a stable degree $(1, d)$ map $\psi: C \rightarrow \mathbb{C} P^{1} \times \mathbb{C} P^{N-1}$. There exist a unique irreducible component $C_{0} \in C$ such that $\left.\psi\right|_{C_{0}}$ has degree $\left(1, d^{\prime}\right)$ where $d^{\prime} \leq$ $d$. The image $\psi\left(C_{0}\right)$ is the graph of a map $\mathbb{C} P^{1} \rightarrow \mathbb{C} P^{N-1}$ of degree $d^{\prime}$. The map is given by the binary forms $\left(P_{1}: \ldots: P_{N}\right)$ of degree $d^{\prime}$ with no common factors and determines the forms uniquely up to a non-zero constant factor. The curve $C-C_{0}$ has $r$ connected components which are mapped to $\mathbb{C} P^{1} \times \mathbb{C} P^{N-1}$ with degrees $\left(0, d_{1}\right), \ldots,\left(0, d_{r}\right), d_{1}+\ldots+d_{r}=d-d^{\prime}$, and the image of $i$-th component is situated in the slice $\left(a_{i}: b_{i}\right) \times \mathbb{C} P^{N-1}$. We put $\mu([\psi])=\prod_{i=1}^{r}\left(a_{i} \xi-b_{i} \zeta\right)^{d_{i}}\left(P_{1}: \ldots: P_{r}\right)$.

Proposition 2.1 ( see the Main Lemma in [10]). The Aut $\left(\mathbb{C} P^{1}\right)$-equivariant map $\mu: G_{d}\left(\mathbb{C} P^{N-1}\right) \rightarrow L_{d}$ is regular.

For a compact projective submanifold $X \subset \mathbb{C} P^{N-1}$ the graph space $G_{d}(X)$ is embedded into the space $G_{D}\left(\mathbb{C} P^{N-1}\right)$ where $D=p(d)$ is the intersection index of the class $p \in H^{2}(X)$ of hyperplane sections with the fundamental class of degree $d$ curves. Consider the hyperplane line bundle over the projective space $L_{D}$ and induce it to $G_{d}(X)$ by the restriction of the map $\mu$. We will call it the universal line bundle corresponding to the embedding of $X$ to $\mathbb{C} P^{N-1}$. The universal line bundle is $A u t\left(\mathbb{C} P^{1}\right)$-equivariant.

Let now $X$ be a compact projective manifold provided with a holomorphic action of a compact Lie group $G$. Let us assume for simplicity that the 1-st Chern class identifies the Picard group of $X$ with $H^{2}(X)$. Represent a basis in $H^{2}(X)$ over $\mathbb{Q}$ by the 1 -st Chern classes of very ample $G$-equivariant line bundles over $X$. The embeddings of $X$ to projective spaces defined by holomorphic sections of these line bundles are $G$-equivariant. The universal line bundles over $G_{d}(X)$ corresponding to these embeddings are $A u t\left(\mathbb{C} P^{1}\right) \times G$ equivariant in this case. We can now extend the construction of universal line bundles from ample to arbitrary elements of the Picard group by additivity. For a given equivariant line bundle over $X$ with the $G$-equivariant 1-st Chern class $p \in H_{G}^{2}(X)$ we will denote with the same letter $p$ the $\operatorname{Aut}\left(\mathbb{C} P^{1}\right) \times G$ equivariant 1-st Chern class of the corresponding universal line bundle over $G_{d}(X)$. We will call $p \in H_{P S L_{2}(\mathbb{C}) \times G}^{2}\left(G_{d}(X)\right)$ the universal class correspond- 
ing to $p \in H_{G}^{2}(X)$.

In the sequel we will use $S^{1}$-equivariant cohomology with respect to the maximal torus $S^{1} \subset P S L_{2}(\mathbb{C})$. We will use the lifting of this action to $\mathbb{C}^{2}$ and to the spaces of binary forms defined in the way breaking the $P S L_{2}(\mathbb{C})$ symmetry:

$$
(\zeta, \xi) \mapsto(\exp (2 \pi i \phi) \zeta, \xi)
$$

If $-p$ denotes the equivariant 1 -st Chern class of the $S^{1}$-equivariant Hopf bundle defined by this lifting, then the equivariant integration over $\mathbb{C} P^{1}$ and $L_{D}$ is described respectively by the residue formulas

$$
\begin{gathered}
f(p, \hbar) \mapsto \frac{1}{2 \pi i} \oint \frac{f(p, \hbar) d p}{p(p+\hbar)}, \\
f(p, \hbar) \mapsto \frac{1}{2 \pi i} \oint \frac{f(p, \hbar) d p}{p(p-\hbar)(p-2 \hbar) \ldots(p-D \hbar)},
\end{gathered}
$$

where $\mathbb{Z}[\hbar]=H^{*}\left(\mathbb{C} P^{\infty}\right)=H^{*}\left(B S^{1}\right)$ stands for the coefficient ring of the $S^{1}$-equivariant cohomology theory.

Remark. The graph spaces can be considered as approximations to the space of loops in $X$. In this interpretation the universal classes correspond to $S^{1}$-equivariant forms

$$
\text { (symplectic structure) }+\hbar \text { (action functional) }
$$

on the loop space. Though heuristicly important, such a relation with the Floer theory on the loop space (see [8]) is technically avoidable. The same applies to Proposition 2.1 (see the remark after Proposition 4.1).

3. Symplectic toric manifolds. Consider the standard real symplectic space $\mathbb{C}^{N}$ provided with the symplectic structure $\operatorname{Im} \sum d \bar{z}_{j} \wedge d z_{j} / 2$. The maximal torus $T^{N}$ acts on $\mathbb{C}^{N}$ by linear symplectic transformations $z \mapsto$ $\operatorname{diag}\left(\exp 2 \pi i x_{1}, \ldots, \exp 2 \pi i x_{N}\right) z$. The momentum map $J$ of this action maps $\mathbb{C}^{N}$ onto the closed 1-st orthant $\mathbb{R}_{+}^{N}$ in Lie $^{*} T^{N}$.

Consider a subtorus $T^{k} \subset T^{N}$. The momentum map of the action $T^{k}$ : $\mathbb{C}^{N}$ is the composition of $J$ with the linear integral projection $M: \mathbb{R}_{+}^{N} \rightarrow$ $\mathbb{R}^{k}=L i e^{*} T^{k}$. Pick a regular value $t$ of the momentum map and define the symplectic orbifold $X$ as the symplectic reduction $X=\mathbb{C} / /{ }_{t} T^{k}=(M \circ$ $J)^{-1}(t) / T^{k}$. 
Denote $K \subset \mathbb{R}^{k}$ the connected component containing $t$ of the regular value set. The orbifold $X$ is canonically identified with the complex quotient $(M \circ J)^{-1}(K) / T_{\mathbb{C}}^{k}$. This quotient is a Gorenstein projective toric variety of dimension $N-k$. In particular, it has no codimension 1 singularities.

The symplectic form on $X$ depends on the choice of the momentum value $t \in K$ and is Kähler with respect to the complex structure. The action of the quotient torus $T^{N} / T^{k}$ on $X$ preserves the form. The momentum polyhedron of this action is the fiber $M^{-1}(t)$ in the orthant $\mathbb{R}_{+}^{N}$. The orbifold $X$ is compact if and only if the polyhedron is compact or, equivalently, if $M^{-1}(0)=\{0\}$. If it is the case, we call $X$ a compact symplectic toric variety.

Vice versa, a compact projective toric variety $X$ with at most Gorenstein singularities can be obtained as the above symplectic reduction $\mathbb{C}^{N} / /{ }_{t} T^{k}$ with $N$ equal to the number of hyperplane walls of the the momentum polyhedron. The polyhedron is described by $N$ inequalities $H_{j} \geq c_{j}$ where $H_{j}$ are reduced integral linear functions on $\operatorname{Lie}^{*} T^{N-k}$. These functions define an embedding of the polyhedron onto a section of the 1-st orthant in $\mathbb{R}^{N}$.

In the sequel we will assume that $X$ is a compact symplectic toric variety $\mathbb{C} / /{ }_{t} T^{k}$ with the number of hyperplane walls of the momentum polyhedron equal to $N$. We review below some basic properties of $X$ (see [1, 7]).

(1) The Gorenstein variety $X$ is non-singular if and only if all the $k$ dimensional faces of the orthant $\mathbb{R}_{+}^{N}$ whose projection to $\mathbb{R}^{k}$ contain $K$ are mapped to $\mathbb{R}^{k}$ with the determinant \pm 1 .

(2) The correspondence between the regular values $t \in K$ of the momentum map and cohomology classes of Kähler symplectic forms is linear and extends to an isomorphism $\mathbb{R}^{k} \rightarrow H^{2}(X, \mathbb{R})$. The isomorphism identifies $K$ with the Kähler cone of $X$, and the Picard group $H^{2}(X, \mathbb{Z})$ - with the lattice $\mathbb{Z}^{k} \subset \mathbb{R}^{k}$ of characters of the torus $T^{k}$. The 1 -st Chern class of the tangent sheaf $\mathcal{T}_{X}$ is represented by the projection $M\left(u_{1}+\ldots+u_{N}\right)$ of the vector $(1, \ldots, 1) \in \mathbb{R}^{N}$.

(3) The cohomology algebra $H^{*}(X, \mathbb{C})$ is multiplicatively generated by Kähler classes and can be described as follows. In the space $\operatorname{Lie} T_{\mathbb{C}}^{N}$ dual to $\mathbb{R}_{\mathbb{C}}^{N}$ consider the union $X^{*}$ of $(N-k)$-dimensional coordinate subspaces - the orthogonal complements to those $k$-dimensional faces of $\mathbb{R}_{+}^{N}$ whose projections to $R^{k}$ contain $K$. Denote $\mathcal{J}$ the ideal of $X^{*}$ in the algebra $\mathbb{C}\left[u_{1}, \ldots, u_{N}\right]$ of regular functions on $\operatorname{Lie}_{\mathbb{C}}^{N}$. In the algebra $\mathbb{C}\left[p_{1}, \ldots, p_{k}\right]$ of regular functions on $\operatorname{Lie}_{\mathbb{C}}^{k}$ consider the ideal $\mathcal{I}$ induced from $\mathcal{J}$ by the embedding $\operatorname{Lie}_{\mathbb{C}}^{k} \subset \operatorname{Lie} T_{\mathbb{C}}^{N}$. Then $H^{*}(X, \mathbb{C})$ is canonically isomorphic to 
$\mathbb{C}[p] / \mathcal{I}$. The isomorphism is induced by the correspondence between the infinitesimal characters $u_{j}$ and 1-st Chern classes of invertible sheaves on $X$. This description is valid over $\mathbb{Q}$, and for non-singular $X-$ over $\mathbb{Z}$.

(4) The algebra $\mathbb{C}[u] / \mathcal{J}$ of regular functions on $X^{*}$ is similarly identified with the equivariant cohomology algebra of $X$ with respect to the action of the quotient torus $T^{N} / T^{k}$. By definition, the equivariant cohomology algebra of a $G$-space $Y$ is the cohomology algebra of the homotopic quotient $E G \times_{G}$ $Y$ and has the module structure over the ring $H^{*}(B G)$ of $G$-characteristic classes. In the case of a torus $G$ the ring is identified with the polynomial algebra on LieG. The $\mathbb{C}\left[L i e T^{N} / T^{k}\right]$-module structure on $\mathbb{C}\left[X^{*}\right]$ is defined by the projection of $X^{*}$ along $L i e T^{k}$.

We will use the $T^{N}$-equivariant cohomology algebra $H_{T^{N}}^{*}(X, \mathbb{C})$. Denote $\mathbb{C}\left[\lambda_{1}, \ldots, \lambda_{N}\right]$ another copy of the characteristic class algebra $H^{*}\left(B T^{N}, \mathbb{C}\right)$ which will play the role of the coefficient ring of $T^{N}$-equivariant theory throughout the paper. Denote $\left(m_{i j}\right)_{i=1}^{k}{ }_{j=1}^{N}$ the matrix of the projection $M: \mathbb{R}_{+}^{N} \rightarrow \mathbb{R}^{k}$. The $\mathbb{C}[\lambda]$-algebra $H_{T^{N}}^{*}(X, \mathbb{C})$ is multiplicatively generated by $\left(p_{1}, \ldots, p_{k}\right)$ satisfying the relations $u_{j}=\sum_{i=1}^{k} p_{i} m_{i j}-\lambda_{j}$ where $\left(u_{1}, \ldots, u_{N}\right)$ are the generators of $\mathbb{C}\left[X^{*}\right]=\mathbb{C}[u] / \mathcal{J}$. For example, if $M=(1, \ldots, 1)$ then $X=\mathbb{C} P^{N-1}, \mathcal{J}=\left(u_{1} \ldots u_{N}\right)$, and $H_{T^{N}}^{*}(X, \mathbb{C})$ is isomorphic to $\mathbb{C}[p, \lambda] /((p-$ $\left.\left.\lambda_{1}\right) \ldots\left(p-\lambda_{N}\right)\right)$. Here $-p$ represents the equivariant 1-st Chern class of the Hopf bundle over $\mathbb{C} P^{N}$ provided with a natural lift of the torus action. In general equivariant 1-st Chern classes of $T^{N}$-equivariant invertible sheaves on $X$ are represented by integral linear combinations of $u_{j}$. In particular, $u_{1}+\ldots+u_{N}$ represents the equivariant anti-canonical class of $X$.

(5) Integration over the fundamental cycle defines an $H^{*}(B G)$-linear functional with values in $H^{*}(B G)$ and the corresponding $H^{*}(B G)$-bilinear Poincare pairing $\langle f, g\rangle=\int_{X} f \wedge g$ on the equivariant cohomology algebra of a compact $G$-manifold. The Borel fixed point localization theorem gives rize to the following explicit description of such integration in the case of the toric symplectic variety $X$ :

$$
\int_{X} f(p, \lambda)=\sum_{\alpha} \operatorname{Res}_{\alpha} \frac{f(p, \lambda) d p_{1} \wedge \ldots \wedge d p_{k}}{u_{1}(p, \lambda) \ldots u_{N}(p, \lambda)} .
$$

Here the index $\alpha$ runs the set of fixed points of the torus action on $X$. The fixed points correspond to the vertices of the momentum polyhedron $M^{-1}(t)$ and therefore - to those $k$-faces of $\mathbb{R}_{+}^{N}$ whose projections to $\mathbb{R}^{k}$ 
contain $t$. The orthogonal complement of such a face is given by $k$ equations $u_{j_{1}}=\ldots=u_{j_{k}}=0, j_{1}<\ldots<j_{k}$. The symbol $\operatorname{Res}_{\alpha}$ refers to the residue of the $k$-form at the pole specified by the ordered set of equations

$$
\sum_{i=1}^{k} p_{i} m_{i j_{s}}=\lambda_{s}, s=1, \ldots, k .
$$

Permutations of the equations affect the sign of the residue.

We will denote $p(\alpha)=\left(p_{1}(\alpha), \ldots, p_{k}(\alpha)\right)$ the solution to this system of linear equations and $u_{j}(\alpha)$ the values of the linear functions $u_{j}=\sum p_{i} m_{i j}-\lambda_{j}$ at the point $p(\alpha)$. We will also identify in our notations the index $\alpha$ of the fixed point of $T^{N}: X$ with the corresponding vertex of the momentum polyhedron and with the set $\left\{j_{1}, \ldots, j_{k}\right\}$.

Although the residues on the RHS are rational functions of $\lambda$, their sum is a polynomial provided that $f \in H_{T^{N}}^{*}(X, \mathbb{C})$ is represented by a polynomial $f(p, \lambda)$. The equivariant Poincare pairing $\langle f, g\rangle=\int_{X} f g$ is non-degenerate over $\mathbb{Q}[\lambda]$ (and even $\mathbb{Z}[\lambda]$ if $X$ is non-singular). The value at $\lambda=0$ of the LHS represents $\int_{X} f(p, 0)$ and gives rise to the ordinary Poincare pairing on $H^{*}(X)$.

Notice that the toric variety $X$ and its properties have been described entirely in terms of the $N$ integer vectors $M u_{1}, \ldots, M u_{N}$ in $\mathbb{R}^{k}$ and the chosen Kähler cone $K \subset \mathbb{R}^{k}$.

We will assume further on that $X$ denotes a non-singular compact symplectic toric manifold.

A codimension $l$ complete intersection $Y \subset X$ is by definition the common zero locus of holomorphic sections of $l$ line bundles. Let $v_{1}, \ldots, v_{l}$ be the infinitesimal characters of $T^{N}$ which specify $l$ equivariant line bundles $\mathcal{L}_{a}$. We will assume that $\mathcal{L}_{a}$ are non-negative, i.e. $M v_{a} \in \bar{K}$, and therefore that the bundle $\mathcal{V}=\oplus_{a} \mathcal{L}_{a}$ is convex.

We provide the bundle $\mathcal{V}$ with the additional action of the $l$-dimensional torus $T^{l}$ acting fiberwise by scalar multiplication in each summand $\mathcal{L}_{a}$. We will study the equivariant Gromov - Witten theory of the pair $(X, \mathcal{V})$ with respect to the action of the torus $T=T^{N} \times T^{l}$. The algebra

$$
\mathbb{Q}\left[\lambda_{1}, \ldots, \lambda_{N}, \lambda_{1}^{\prime}, \ldots, \lambda_{l}^{\prime}\right]=H^{*}(B T, \mathbb{Q})
$$

will play the role of the coefficient algebra of the theory. Now on we will assume that $u_{j}, v_{a}, p_{i}$ denote corresponding $T$-equivariant cohomology classes. 
In particular the $T$-equivariant 1 -st Chern classes of the line bundles $\mathcal{L}_{a}$ and their fixed point localizations are represented by the linear combinations $v_{a}:=\sum l_{i a} p_{i}-\lambda_{a}^{\prime}$, and $v_{a}(\alpha)=\sum l_{i a} p_{i}(\alpha)-\lambda_{a}^{\prime}$.

We introduce the $T$-equivariant integration over the virtual fundamental class $[Y]$ of the corresponding (invariant) complete intersections:

$$
\int_{[Y]} f:=\int_{X} f \operatorname{Euler}(\mathcal{V})=\sum_{\alpha} \operatorname{Res}_{\alpha} f\left(p, \lambda, \lambda^{\prime}\right) \frac{v_{1} \ldots v_{l} d p_{1} \wedge \ldots \wedge d p_{k}}{u_{1} \ldots u_{N}} .
$$

In the non-equivariant limit $\lambda=0, \lambda^{\prime}=0$ it reduces to the integration over the fundamental class of non-singular comlete intersections $Y \subset X$ as well as the whole Gromov-Witten theory - to that for $Y$, according to the axiom (7).

Degrees of compact holomorphic curves in $X$ form the semigroup

$$
\Lambda=\left\{d \in H_{2}(X) \mid(t, d) \geq 0 \forall t \in \bar{K}\right\} .
$$

The Kahler cone $\bar{K}$ is the intersection $\cap_{\alpha} \Delta_{\alpha}$ of of images in $\mathbb{R}^{k}$ of $k$-faces in $\mathbb{R}_{+}^{N}$. Respectively, $\Lambda$ coincides with the convex hull of the union $\cup_{\alpha} \Delta_{\alpha}^{*}$ of the orthants $\Delta_{\alpha}^{*} \subset \mathbb{R}^{k *}$ polar to the orthants $\Delta_{\alpha}$. We will denote $\mathbb{Z}[[\Lambda]]$ the formal completion of the semigroup ring $\mathbb{Z}[\Lambda]$ and represent an element $d \in \Lambda$ by the monomial $q^{d}=q_{1}^{d_{1}} \ldots q_{k}^{d_{k}} \in \mathbb{Z}[\Lambda]$ where $\left(d_{1}, \ldots, d_{k}\right)$ are coordinates of $d$ in $\left(\mathbb{Z}^{k}\right)^{*}$.

We will write $d \geq d^{\prime}$ if $d-d^{\prime} \in \Lambda$. It is a partial order on $H_{2}(X)$.

We will also use the notations

$$
D_{j}=\sum_{i} d_{i} m_{i j}, L_{a}=\sum_{i} l_{i a} d_{i}
$$

(which mask the actual dependence of these integers on the vector $d$ ).

We complete this section with some notations and elementary information about the 1-dimensional orbits of $T_{\mathbb{C}}^{N}: X$ which will be exploited, in accordance with the axiom (8), in the fixed point localization technique.

The 1-dimensional orbits correspond to the 1-dimensional edges of the momentum polyhedron. The vertex $\alpha$ of the momentum polyhedron $M^{-1}(t)$ is situated in the $k$-face $\left\{\left(T_{1}, \ldots, T_{N}\right) \mid T_{s}=0, \forall s \notin \alpha\right\}$ of $\mathbb{R}_{+}^{N}$ and connected by one-dimensional edges to $N-k$ other vertices $\beta(\alpha, j), j \notin \alpha$, situated in the $(k+1)$-faces $\left\{\left(T_{1}, \ldots, T_{N}\right) \mid T_{s}=0 \forall s \notin \alpha, s \neq j\right\}$. The edge itself 
is the momentum polyhedron of the closure of a 1-dimensional toric orbit $\mathbb{C}^{k+1} / /{ }_{t} T^{k}$ in $X$ isomorphic to $\mathbb{C} P^{1}$. We denote $d(\alpha, j) \in \Lambda$ the degree of this $\mathbb{C} P^{1}$ in $X$. The corresponding $D_{s}(\alpha, j):=\sum d_{i}(\alpha, j) m_{i s}$ vanish for $k-1$ values of the index $s \in \alpha \cap \beta(\alpha, j)$ and are equal to 1 for the two values of $s \in(\alpha \triangle \beta)=\left\{j, j^{\prime}\right\}$. We denote $L_{a}(\alpha, j)$ the corresponding values $\sum d_{i}(\alpha, j) l_{i a}$ of $L_{a}$. Notice that $\beta$ and $j^{\prime} \in \alpha$ depend on and are uniquely determined by a choice of $\alpha$ and $j \notin \alpha$.

For $\beta=\beta(\alpha, j)$ we have $d(\alpha, j)=d\left(\beta, j^{\prime}\right)$ and

$$
u_{s}(\alpha)=u_{s}(\beta)+D_{s}(\alpha, j) u_{j}(\alpha), v_{a}(\alpha)=v_{a}(\beta)+L_{a}(\alpha, j) u_{j}(\alpha),
$$

In particular, $u_{j}(\alpha)=-u_{j^{\prime}}(\beta)$.

4. The generating function. Let $(X, \mathcal{V})$ be the nonsingular compact toric symplectic manifold provided with the convex bundle equivariant with reapect to the action of $T=T^{N} \times T^{l}$ as described in the previous section. Denote $\mathcal{V}_{d}$ the vector bundle over $G_{d}(X)$ with the fiber $H^{0}\left(C, \psi^{*} \pi_{2}^{*} \mathcal{V}\right)$ over the point represented by the stable map $\phi: C \rightarrow \mathbb{C} P^{1} \times X$. We will study the following series:

$$
\mathcal{G}\left(z, q, \hbar ; \lambda, \lambda^{\prime}\right)=\sum_{d \in \Lambda} q^{d} \int_{\left[G_{d}(X)\right]} e^{P_{1} z_{1}+\ldots+P_{k} z_{k}} \operatorname{Euler}\left(\mathcal{V}_{d}\right) .
$$

Here $\left(P_{1}, \ldots, P_{k}\right)$ denote the universal classes in $H_{T \times S^{1}}^{2}\left(G_{d}(X), \mathbb{Q}\right)$ corresponding to the $T$-equivariant classes $p_{i} \in H_{T}^{2}(X)$.

Proposition 4.1. The series $\mathcal{G}=\sum g_{d, m} q^{d} z^{m}$ has polynomial coefficients $g_{d, m} \in \mathbb{Q}\left[\hbar, \lambda, \lambda^{\prime}\right]$.

Proof. The equivariant cohomology classes $P_{i}$ and Euler $\left(\mathcal{V}_{d}\right)$ of $G_{d}(X)$ are defined over $\mathbb{Q}\left[\hbar, \lambda, \lambda^{\prime}\right]$, and the integration over the Gromov-Witten class $\left[G_{d}(X)\right]$ assumes values in $\mathbb{Q}\left[\hbar, \lambda, \lambda^{\prime}\right]$ by the axiom $(1)$.

Remark. The polynomiality property of $g_{d, m}$ is crucial for our proof of Theorems $0.1,0.2$. However one can define the series $\mathcal{G}$ in terms of GromovWitten theory on $\mathbb{C} P^{1} \times X$ (see [10], Section 6) without mentioning the universal classes $P_{i}$, and the polynomial property then follows directly from the axiom (1). Thus our use of Proposition 2.1 is avoidable.

Proposition 4.2. (see [10).

$$
\mathcal{G}(z, q, \hbar)=\int_{[Y]} \mathcal{S}(q \exp (\hbar z), \hbar) e^{p z} \mathcal{S}(q,-\hbar)
$$


where $\mathcal{S}\left(q, \hbar ; \lambda, \lambda^{\prime}\right)$ is determined from the condition that for any $a \in H_{T}^{*}(X)$

$$
\int_{[X]} \mathcal{S} a=\int_{[X]} \operatorname{Euler}(\mathcal{V}) a+\sum_{d \in \Lambda-0} q^{d} \int_{\left[X_{2, d}\right]} \frac{\operatorname{ev}_{1}^{*}(a) \operatorname{Euler}\left(\mathcal{V}_{2, d}\right)}{(\hbar-c)} .
$$

Proof. The proposition is easily deduced (see [10]) by localization to fixed points of the $S^{1}$-action on $G_{d}(X)$ and $L_{D}$ and from the following corollary of the axiom (2):

$$
\int_{\left[X_{2, d}\right]} \frac{\operatorname{fgt}_{2}^{*}(A)}{\hbar-c}=\int_{\left[X_{1, d}\right]} \frac{A}{\hbar(\hbar-c)} .
$$

Corollary 4.3. $\mathcal{S}=1+o(1 / \hbar)$ when written as a formal power series in $\hbar^{-1}$

Proof: $\operatorname{Euler}\left(\mathcal{V}_{2, d}\right)=\operatorname{fgt}_{2}^{*}\left(\operatorname{Euler}\left(\mathcal{V}_{1, d}\right)\right)$.

Remark. The vector-function $J_{\mathcal{V}}$ in Theorem 0.2 is defined in Section 1 as $\mathcal{S} \exp \left(\left(t_{0}+\sum P_{i} \log q_{i}\right) / \hbar\right)$.

Denote $\mathcal{S}_{\alpha}$ the restriction of the $T$-equivariant cohomology class $\mathcal{S}=$ $\sum_{d \in \Lambda} S_{\alpha, d} q^{d}$ to the fixed point $\alpha$ of the torus $T$ action on $X$. The coefficients $\mathcal{S}_{\alpha, d}$ of the series $\mathcal{S}_{\alpha}$ are rational functions of $\left(\hbar, \lambda, \lambda^{\prime}\right)$.

Proposition 4.4. The series $\mathcal{S}_{\alpha}$ satisfy the following recursion relations:

$$
\begin{gathered}
\mathcal{S}_{\alpha}\left(q, \hbar ; \lambda, \lambda^{\prime}\right)=\sum_{d \in \Lambda} R_{\alpha, d}\left(\hbar^{-1} ; \lambda, \lambda^{\prime}\right) q^{d}+ \\
\sum_{j \notin \alpha} \sum_{n=1}^{\infty} q^{n} d_{\alpha, j} \frac{C_{\alpha, j}(n)}{n \hbar+u_{j}(\alpha)} \mathcal{S}_{\beta(\alpha, j)}\left(q,-u_{j}(\alpha) / n ; \lambda, \lambda^{\prime}\right)
\end{gathered}
$$

where $R_{\alpha, d}$ are polynomials in $\hbar^{-1}$ with coefficients in $\mathbb{Q}\left(\lambda, \lambda^{\prime}\right)$, and the recursion coefficients $C_{\alpha, j}(n)=$

$$
\frac{\left.\Pi_{a} \Pi_{m=1}^{n L_{a}(\alpha, j)}\left(v_{a}(\alpha)-m u_{j}(\alpha) / n\right) \Pi_{s \notin \alpha \cup \beta} \Pi_{m \leq 0}\left(u_{s}(\alpha)-m u_{j}(\alpha) / n\right)\right)}{\left.(n-1) !\left(u_{j}(\alpha) / n\right)^{n-1}\right) \Pi_{s \notin \beta} \Pi_{m \leq n D_{s}(\alpha, j)}\left(u_{s}(\alpha)-m u_{j}(\alpha) / n\right)} .
$$

Proof. We apply the fixed point localization technique to the action of the torus $T$ on $X_{2, d}$. Consider a fixed point represented by the stable map $f:\left(C, x_{1}, x_{2}\right) \rightarrow X$ and denote $C^{\prime}$ the irreducible component of $C$ carrying 
the marked point $x_{1}$. The contribution of this fixed point to $\mathcal{S}_{\alpha}$ via the localization formula described in the axiom (8) vanishes unless $f\left(x_{1}\right)=\alpha$.

If $f\left(C^{\prime}\right)=\alpha$, then the contribution involves integration over the space $\overline{\mathfrak{M}}_{r}$ of configurations of $r>2$ special points on the connected component of $f^{-1}(\alpha)$ containing $x_{1}$. The universal cotangent line at the 1 -st marked point localizes to the line bundle over $\overline{\mathfrak{M}}_{r}$ formed by the cotangent lines to $C^{\prime}$ at $x_{1}$. Since the action of $T$ on this line bundle is trivial, the localization of the Chern class $c$ is nilpotent. Thus the contribution of such fixed points to the localization formula is polynomial in $1 / \hbar$.

If $f\left(x_{1}\right)=\alpha$ but $f\left(C^{\prime}\right) \neq \alpha$ then $f^{\prime}:=\left(f \mid C^{\prime}\right)$ is the degree $n$ cover of a 1-dimensional orbit of $T_{\mathbb{C}}$ in $X$ connecting the fixed point $\alpha$ with another fixed point $\beta=\beta(\alpha, j)$. In this case $f$ is glued from $f^{\prime}:\left(C^{\prime}, x_{1}, x\right) \rightarrow X$ and a stable map $f^{\prime \prime}:\left(C^{\prime \prime}, x, x_{2}\right) \rightarrow X$ of degree $d^{\prime \prime}=d-n d(\alpha, j)$ with $f^{\prime}(x)=$ $f^{\prime \prime}(x)=\beta$. The localization of $\hbar-c$ in this case equals $\hbar+u_{j}(\alpha) / n$. The contribution of $T_{x}^{\prime} \otimes T_{x}^{\prime \prime}$ to the Virtual Normal Euler Class in the localization axiom equals $-u_{j}(\alpha) / n-c$ where $c$ is the equivariant 1-st Chern class of the universal cotangent line over $X_{2, d^{\prime \prime}}$ at the marked point $x$. The remaining part of the Virtual Normal Euler Class is the product of such classes for $f^{\prime \prime}$ in $X_{2, d^{\prime \prime}}$ and for $f^{\prime}$ in $X_{2, n d(\alpha, j)}$. The latter one can be easily computed by using the quotient description of the tangent bundle to $X=\mathbb{C}^{N} / / T^{k}$ as a virtual direct sum of line bundles and is equal to $1 / C_{\alpha, j}(n)$. Also we have $|A u t f|=n\left|A u t f^{\prime \prime}\right|$ where $n$ is the order of the cyclic automorphism group of the $n$-fold cover $f^{\prime}: z \mapsto z^{n}$.

The summation over all $d^{\prime \prime}, j \notin \alpha$ and $n=1,2, \ldots$ gives rise to the recursion relation.

Proposition 4.5. The series $\mathcal{S}$ is uniquely determined by the following properties:

(a) the recursion relations of Proposition 4.4,

(b) the asymptotical condition $\mathcal{S}=1+o(1 / \hbar)$ of Corollary 4.3,

(c) the property from Proposition 4.1 of the series

$$
\sum g_{d, m} q^{d} z^{m}=\int_{[Y]} \mathcal{S}\left(q e^{\hbar z}, \hbar\right) e^{p z} \mathcal{S}(q,-\hbar)
$$

to have polynomial coefficients $g_{d, m} \in \mathbb{Q}\left[\hbar, \lambda, \lambda^{\prime}\right]$.

Proof. Consider another solution $\mathcal{S}^{\prime}$ to the same recursion relations (a) with the same polynomials $R_{\alpha, d}^{\prime}=R_{\alpha, d}$ as in $\mathcal{S}$ for all $0 \leq d<d_{0}$ and 
satisfying the asymptotical condition (b). We will show that $R_{\alpha, d_{0}}^{\prime}=R_{\alpha, d_{0}}$. This would imply the uniqueness since, given all the polynomials $R_{a, d}$, the recursion relations (a) allow one to recover the series $\mathcal{S}$ unambiguously.

Consider the $q^{d_{0}}$-term in $\mathcal{G}^{\prime}-\mathcal{G}$. The conditions (a),(b) imply that $\mathcal{S}^{\prime}-\mathcal{S}=$ $R q^{d_{0}}+$ (higher order terms in $q$ ) and that $q^{d_{0}}$-term in $\mathcal{G}^{\prime}-\mathcal{G}$ is equal to

$$
\delta(R)=\int_{[Y]} e^{\left(p+d_{0} \hbar\right) z} R(\hbar, \lambda)+e^{p z} R(-\hbar, \lambda),
$$

where the class $R$ is defined by its localizations $R_{\alpha}=R_{\alpha, d_{0}}^{\prime}-R_{\alpha, d_{0}}$. From (b) and (c) we know that $R$ is a polynomial in $1 / \hbar$ divisible by $1 / \hbar^{2}$ and that the series $\delta=\sum \delta_{m} z^{m}$ has polynomial coefficients $\delta_{m} \in \mathbb{Q}\left[\hbar, \lambda, \lambda^{\prime}\right]$.

A generic value of $\lambda=\left(\lambda_{1}, \ldots, \lambda_{N}\right)$ determines a linear function on the momentum polyhedron $\mathbb{R}_{+}^{N} \cap J^{-1}(z)$ for each $z \in K$ with pairwise distinct values $H_{\alpha}$ at the vertices. Computing $\delta\left(A \hbar^{-1}+B\right)$ modulo $\hbar$ for a generic ray $t \mapsto t z$ we find

$$
\sum_{\alpha} e^{H_{\alpha} t}\left(A_{\alpha} t\left(z, d_{0}\right)+B_{\alpha}\right) \operatorname{Res}_{\alpha} \frac{v_{1} \ldots v_{l} d p_{1} \wedge \ldots \wedge d p_{k}}{u_{1} \ldots u_{N}} .
$$

Here $v_{1}(\alpha) \ldots v_{a}(\alpha) \neq 0$ for generic values of $\lambda^{\prime}$, and $\left(z, d_{0}\right)>0$. Since the functions $\exp \left(H_{\alpha} t\right), t \exp \left(H_{\alpha} t\right)$ with distinct $H \alpha$ are linearly independent, we conclude that $\delta(A / \hbar+B)=o(\hbar)$ implies $A=B=0$ in $H_{T}^{*}\left(X, \mathbb{C}\left(\lambda, \lambda^{\prime}\right)\right)$ for generic and therefore for all values of $\lambda, \lambda^{\prime}$ and $z$. Applying this conclusion to $\hbar^{2 r} R(1 / \hbar, \lambda)$ with $r>0$ and $R(1 / \hbar)=A \hbar^{-2 r-1}+B \hbar^{-2 r}+\ldots$ of degree $\leq(2 r+1)$ we find that $\operatorname{deg} R \leq(2 r-1)$ and by induction - that $\operatorname{deg} R \leq 1$. Now the assumption (b) that $R$ has no terms of order $\hbar^{0}$ and $\hbar^{-1}$ implies that $R=0$.

5. Toric map spaces. In this section we describe toric compactifications of spaces of holomorphic maps $\mathbb{C} P^{1} \rightarrow X$.

For each $d \in \Lambda$ consider the following complex space of $N$-dimensional vector-polynomials in one complex variable $\zeta$ :

$$
\left\{\left(z_{1}(\zeta), \ldots, z_{N}(\zeta)\right) \mid \operatorname{deg} z_{j} \leq D_{j}\right\}
$$

The torus $T^{N}$ acts on this space componentwise. Denote $J_{d}$ the momentum map of the induced action of $T^{k} \subset T^{N}$, pick $t \in K$ and introduce the symplectic toric variety $X_{d}=J_{d}^{-1}(t) / T^{k}$. It is compact and nonsingular 
whenever $X$ is compact and nonsingular. Generic points in $X_{d}$ represent degree $d$ holomorphic maps

$$
\mathbb{C} P^{1} \rightarrow X: \zeta \mapsto\left(z_{1}(\zeta), \ldots, z_{N}(\zeta)\right) \bmod T_{\mathbb{C}}^{k}
$$

The variety $X_{d}$ is empty unless $d \in \cup_{\alpha} \Delta_{\alpha}^{*}$.

The rotation $\zeta \mapsto \exp (2 \pi i \phi) \zeta$ of $\mathbb{C} P^{1}$ induces an $S^{1}$-action on $X_{d}$. Fixed points of the $S^{1} \times T^{N}$-action on $X_{d}$ are isolated. The Borel localization formula for $X_{d}$ yields:

$$
\int_{X_{d}} f(p, \hbar, \lambda)=\sum_{\alpha: d \in \Delta_{\alpha}^{*}} \sum_{r} \operatorname{Res}_{\alpha, r} \frac{f(p, \hbar, \lambda) d p_{1} \wedge \ldots \wedge d p_{k}}{\prod_{j: D_{j} \geq 0}\left(u_{j}\left(u_{j}-\hbar\right)\left(u_{j}-2 \hbar\right) \ldots\left(u_{j}-D_{j} \hbar\right)\right)} .
$$

Here $\operatorname{Res}_{\alpha, r}$ refers to the residue at the point specified by the equations

$$
u_{j_{1}}(p, \lambda)=r_{1} \hbar, \ldots, u_{j_{k}}(p, \lambda)=r_{k} \hbar, j_{1}<\ldots<j_{k},
$$

with $\left\{j_{1}, \ldots, j_{k}\right\}=\alpha$ and $r$ runs the integer vectors

$$
r=\left(r_{1}, \ldots, r_{k}\right): 0 \leq r_{1} \leq D_{j_{1}}, \ldots, 0 \leq r_{k} \leq D_{j_{k}} .
$$

The equivariant cohomology algebra of $X_{d}$ is identified with the quotient of $\mathbb{C}[p, \hbar, \lambda]$ by the kernel ideal of the corresponding Poincare pairing.

The dimension of $X_{d}$ may exceed the Riemann-Roch dimension $\operatorname{dim} X+$ $\left(c_{1}\left(\mathcal{T}_{X}\right), d\right)=N-k+D_{1}+\ldots+D_{N}$ of the space of degree $d$ holomorphic maps $\mathbb{C} P^{1} \rightarrow X$ if some $D_{j}=\sum d_{i} m_{i j}$ are negative. We introduce the virtual fundamental class $\left[X_{d}\right]$ of the Riemann-Roch dimension as the Poincare-dual to the equivariant Euler class of the following vector bundle over $X_{d}$.

Consider the complex space of the vector polynomials in the variable $\zeta^{-1}$ defined by

$$
\left\{\left(z_{1}\left(\zeta^{-1}\right), \ldots, z_{N}\left(\zeta^{-1}\right)\right) \mid z_{j}(0)=0, \operatorname{deg} z_{j}<-D_{j}\right\}
$$

We introduce the $T^{N}$-equivariant locally free sheaf on $X_{d}=J_{d}^{-1}(t) / T^{k}$ associated with the componentwise action of $T^{k} \subset T^{N}$ on this space. The rotation of $\zeta$ defines an $S^{1}$-equivariant structure on the sheaf. At generic points $\psi: \mathbb{C} P^{1} \rightarrow X$ of $X_{d}$ the sheaf coincides with the obstruction sheaf $H^{1}\left(\mathbb{C} P^{1}, \psi^{*}\left(\mathcal{T}_{X}\right)\right)$. 
The Borel localization formula gives rise to the following description of integration over the virtual fundamental class:

$$
\int_{\left[X_{d}\right]} f=\sum_{\alpha: d \in \Delta_{\alpha}^{*}} \sum_{r} \operatorname{Res}_{\alpha, r} d p_{1} \wedge \ldots \wedge d p_{k} f(p, \hbar, \lambda) \Pi_{j=1}^{N} \frac{\Pi_{m=-\infty}^{-1}\left(u_{j}-m \hbar\right)}{\Pi_{m=-\infty}^{D_{j}}\left(u_{j}-m \hbar\right)} .
$$

Consider now the convex bundle $\mathcal{V}=\oplus_{a} \mathcal{L}_{a}$. For a generic degree- $d$ map $\psi: \mathbb{C} P^{1} \rightarrow X$ the space $H^{0}\left(\mathbb{C} P^{1}, \psi^{*} \mathcal{L}_{a}\right)$ can be identified with the space of polynomials $\left\{y(\zeta) \mid \operatorname{deg} y \leq L_{a}\right\}$ where $L_{a}=\sum d_{i} l_{i a}$. We introduce the vector bundle of dimension $L_{a}+1$ over $X_{d}$ associated with the scalar action of $T^{k}$ on this space via the character $\sum l_{i a} p_{i}$. The direct sum of these bundles over $a=1, \ldots, l$ is equivariant with respect to $S^{1} \times T^{N} \times T^{l}$ where $S^{1}$ acts by rotations of $\zeta$, and $T^{l}$ acts by scalr multiplication componentwise on the direct summands. The $S^{1} \times T$-equivariant Euler class of this bundle is $\Pi_{a} v_{a}\left(v_{a}-\hbar\right) \ldots\left(v_{a}-L_{a} \hbar\right)$ where $v_{a}=\sum l_{i a} p_{i}-\lambda_{a}^{\prime}$. We define the virtual fundamental class $\left[Y_{d}\right]$ as the Poincare-dual to this Euler class:

$$
\int_{\left[Y_{d}\right]} f:=\int_{\left[X_{d}\right]} f\left(p, \hbar, \lambda, \lambda^{\prime}\right) \Pi_{a} \Pi_{m=0}^{L_{a}}\left(v_{a}(p, \lambda)-m \hbar\right) .
$$

For polynomial $f \in \mathbb{Q}\left[p, \hbar, \lambda, \lambda^{\prime}\right]$ the integration over $\left[Y_{d}\right]$ assumes polinomial values in $\mathbb{Q}\left[\hbar, \lambda, \lambda^{\prime}\right]$.

6. The hypergeometric series. We will study here the generating function

$$
\Phi(z, q)=\sum_{d \in \Lambda} q^{d} \int_{\left[Y_{d}\right]} e^{p_{1} z_{1}+\ldots+p_{k} z_{k}}
$$

which is a formal power series in $z$ with coefficients in the formal completion of the semigroup ring of $\Lambda$ over the polynomial algebra $\mathbb{Q}\left[\hbar, \lambda, \lambda^{\prime}\right]$.

Proposition 6.1. The series $\Phi$ is weighted-homogeneous of degree $l+$ $k-N$ with respect to the grading

$$
\operatorname{deg} z_{i}=-1, \operatorname{deg} \hbar=\operatorname{deg} \lambda_{j}=\operatorname{deg} \lambda_{a}^{\prime}=1, \operatorname{deg} q_{i}=\sum_{j} m_{i j}-\sum_{a} l_{i a} .
$$

Proof: This follows from grading in equivariant cohomology and the formula $N-k-l+\left(c_{1}\left(\mathcal{T}_{X}\right), d\right)-\left(c_{1}(\mathcal{V}), d\right)$ for the complex dimension of the virtual fundamental cycle $\left[Y_{d}\right]$. 
Consider the formal $q$-series $\Psi$ (which differs from $I_{\mathcal{V}}$ in Theorem 0.2 by the exponential factor $\exp \left(\left(t_{0}+p \log q\right) / \hbar\right)$ only):

$$
\Psi\left(q, \hbar ; \lambda, \lambda^{\prime}\right)=\sum_{d \in \Lambda} q^{d} \frac{\Pi_{a} \Pi_{m \leq L_{a}}\left(v_{a}+m \hbar\right) \Pi_{j} \Pi_{m \leq 0}\left(u_{j}+m \hbar\right)}{\Pi_{a} \Pi_{m \leq 0}\left(v_{a}+m \hbar\right) \Pi_{j} \Pi_{m \leq D_{j}}\left(u_{j}+m \hbar\right)},
$$

where $u_{j}=\sum p_{i} m_{i j}-\lambda_{j}$ and $v_{a}=\sum p_{i} l_{i a}-\lambda_{a}^{\prime}$ are $T$-equivariant cohomology classes of $X$, the integers $D_{j}=\sum d_{i} m_{i j}, L_{a}=\sum d_{i} l_{i a}$ depend on $d=$ $\left(d_{1}, \ldots, d_{k}\right)$, and $m$ runs integer values starting from $-\infty$. Coefficients of the series $\Psi$ are well-defined equivariant cohomology classes of $X$ over the field $\mathbb{C}(\hbar, \lambda)$ of rational functions, and the whole series can be considered as an equivariant cohomology class of $X$ with appropriate coefficients. Notice that a $q^{d}$-term in $\Psi$ has zero localization at the fixed point $\alpha$ unless $d \in \Delta_{\alpha}^{*}$.

Proposition 6.2 (compare with [8, 9, 10]).

$$
\int_{[Y]} \Psi\left(q \exp (\hbar z), \hbar ; \lambda, \lambda^{\prime}\right) e^{p z} \Psi\left(q,-\hbar ; \lambda, \lambda^{\prime}\right)=\Phi\left(z, q, \hbar, \lambda, \lambda^{\prime}\right) .
$$

In particular, $\Phi\left((t-\tau) / \hbar, e^{\tau}, \hbar\right)$ is invariant under the change $(t, \tau, \hbar) \mapsto$ $(\tau, t,-\hbar)$.

Proof. We have

$$
\begin{gathered}
\int_{[Y]} \Psi(q \exp (\hbar z), \hbar) e^{p z} \Psi(q,-\hbar)= \\
\sum_{\alpha} \sum_{d^{\prime}, d^{\prime \prime} \in \Delta_{\alpha}^{*}} q^{d^{\prime}+d^{\prime \prime}} \operatorname{Res}_{\alpha} e^{\left(p+\hbar d^{\prime}\right) z} d p_{1} \wedge \ldots \wedge d p_{k} \times \\
\frac{\prod_{a} \Pi_{m=-L_{a}^{\prime \prime}}^{L_{a}^{\prime}}\left(v_{a}+m \hbar\right) \Pi_{j}\left[\Pi_{m \leq 0}\left(u_{j}+m \hbar\right) \Pi_{m \geq 0}\left(u_{j}+m \hbar\right)\right]}{\Pi_{j} u_{j}\left[\Pi_{m \leq D_{j}^{\prime}}\left(u_{j}+m \hbar\right) \Pi_{m \geq-D_{j}^{\prime \prime}}\left(u_{j}+m \hbar\right)\right]} \\
=\sum_{\alpha} \sum_{d \in \Delta_{\alpha}^{*}} q^{d} \sum_{r} \operatorname{Res}_{\alpha, r} e^{p z} d p_{1} \wedge \ldots \wedge d p_{k} \times \\
\frac{\Pi_{a} \Pi_{m=-L_{a}}^{0}\left(v_{a}+m \hbar\right) \Pi_{j} u_{j} \Pi_{m \in \mathbb{Z}}\left(u_{j}+m \hbar\right)}{\Pi_{j} u_{j}\left[\Pi_{m \leq 0}\left(u_{j}+m \hbar\right) \Pi_{m \geq-D_{j}}\left(u_{j}+m \hbar\right)\right.} \\
=\sum_{\alpha} \sum_{d \in \Delta_{\alpha}^{*}} \sum_{r} \operatorname{Res}_{\alpha, r} \frac{e^{p z} d p_{1} \wedge \ldots \wedge d p_{k} \Pi_{a} \Pi_{m=0}^{L_{a}}\left(v_{a}-m \hbar\right) \Pi_{j} \Pi_{m<0}\left(u_{j}-m \hbar\right)}{\Pi_{j} \Pi_{m \leq D_{j}}\left(u_{j}-m \hbar\right)}
\end{gathered}
$$




$$
=\sum_{d \in \Lambda} q^{d} \int_{\left[Y_{d}\right]} e^{p z}
$$

Consider the localizations $\Psi_{\alpha}\left(q, \hbar ; \lambda, \lambda^{\prime}\right)$ of the class $\Psi$ at the fixed points:

$$
\Psi_{\alpha}=\sum_{d \in \Delta_{\alpha}^{*}} q^{d} \frac{\Pi_{a} \Pi_{m=1}^{L_{a}}\left(v_{a}(\alpha)+m \hbar\right)}{\Pi_{j \in \alpha} D_{j} ! \hbar^{D_{j}}} \Pi_{j \notin \alpha} \frac{\Pi_{m \leq 0}\left(u_{j}(\alpha)+m \hbar\right)}{\Pi_{m \leq D_{j}}\left(u_{j}(\alpha)+m \hbar\right)} .
$$

Each $q^{d}$-term in this series is a rational function of $\hbar$ and can be uniquely written as the sum of simple fractions with poles at $\hbar=-u_{j}(\alpha) / n, j \notin$ $\alpha, 0<n \leq D_{j}$ plus a Laurent polynomial in $\hbar$.

Proposition 6.3. The series $\Psi_{\alpha}$ satisfy the following recursion relations:

$$
\begin{gathered}
\Psi_{\alpha}\left(q, \hbar ; \lambda, \lambda^{\prime}\right)=\sum_{d \in \Delta_{\alpha}^{*}} q^{d} R_{\alpha, d}\left(\hbar, \lambda, \lambda^{\prime}\right)+ \\
\sum_{j \notin \alpha} \sum_{n>0} q^{n d(\alpha, j)} \frac{C_{\alpha, j}(n)}{\left(u_{j}(\alpha)+n \hbar\right)} \Psi_{\beta}\left(q,-u_{j}(\alpha) / n ; \lambda, \lambda^{\prime}\right),
\end{gathered}
$$

where $\beta=\beta(\alpha, j)$ and the coefficients $C_{\alpha, j}(n)=$

$$
\frac{\prod_{a} \Pi_{m=1}^{n L_{a}(\alpha, j)}\left(v_{a}(\alpha)-m u_{j}(\alpha) / n\right) \Pi_{s \notin \alpha \cup \beta} \Pi_{m \leq 0}\left(u_{s}(\alpha)-m u_{j}(\alpha) / n\right)}{(n-1) !\left(u_{j}(\alpha) / n\right)^{n-1} \Pi_{s \notin \beta} \Pi_{m \leq n D_{s}(\alpha, j)}\left(u_{s}(\alpha)-m u_{j}(\alpha) / n\right)} .
$$

Proof. For generic $\lambda, \lambda^{\prime}$ and $\beta=\beta(\alpha, j)$ denote $Z_{\beta}^{d}$ the coefficient of the series $\Psi_{\beta}$ at $q^{d}$. The value of this coefficient at $\hbar=-u_{j}(\alpha) / n=u_{j^{\prime}}(\beta) / n$ is equal to

$$
\frac{\prod_{a} \Pi_{m=1} L_{a}\left(v_{a}(\beta)-m u_{j}(\alpha) / n\right) \Pi_{s} \Pi_{m \leq 0}\left(u_{s}(\beta)-m u_{j}(\alpha) / n\right)}{\Pi_{s} \Pi_{m \leq D_{s}}\left(u_{s}(\beta)-m u_{j}(\alpha) / n\right)}
$$

It vanishes unless $d \in \Delta^{*}(\beta)$ and $D_{j^{\prime}}+n \geq 0$. Due to the relations $u_{s}(\beta)=u_{s}(\alpha)-D_{s}(\alpha, j) u_{j}(\alpha), v_{a}(\beta)=v_{a}(\alpha)-L_{a}(\alpha, j) u_{j}(\alpha)$ we have $Z_{\beta}^{d}\left(-u_{j}(\alpha) / n\right)=$

$$
\frac{\Pi_{a} \Pi_{m>n L_{a}(\alpha, j)}^{L_{a}+n L_{a}(\alpha, j)}\left(v_{a}(\alpha)-m u_{j}(\alpha) / n\right) \Pi_{s} \Pi_{m \leq n D_{s}(\alpha, j)}\left(u_{s}(\alpha)-m u_{j}(\alpha) / n\right)}{\Pi_{s} \Pi_{m \leq D_{s}+n D_{s}(\alpha, j)}\left(u_{s}(\alpha)-m u_{j}(\alpha) / n\right)} .
$$


Since $D_{s}(\alpha, j)=0$ for $s \in \alpha \cap \beta$ and $D_{j}(\alpha, j)=D_{j^{\prime}}(\alpha, j)=1$, we have $C_{\alpha, j}(n) Z_{\beta}^{d}\left(-u_{j}(\alpha) / n\right)=$

$$
\frac{\Pi_{a} \Pi_{m=1}^{L_{a}+n L_{a}(\alpha, j)}\left(v_{a}(\alpha)-\frac{m}{n} u_{j}(\alpha)\right) \Pi_{s \neq j, j^{\prime}} \Pi_{m \leq 0}\left(u_{s}(\alpha)-\frac{m}{n} u_{j}(\alpha)\right)}{\prod_{m=1}^{D_{j^{\prime}}+n}\left(\frac{-n-m}{n} u_{j}(\alpha)\right) \Pi_{m=1}^{D_{j}+n} \quad m \neq n\left(\frac{n-m}{n} u_{j}(\alpha)\right) \prod_{s \neq j, j^{\prime}} \prod_{m \leq D_{s}+n D_{s}(\alpha, j)}\left(u_{s}(\alpha)-\frac{m}{n} u_{j}(\alpha)\right)} .
$$

The last product is exactly the residue of the $q^{d+n d(\alpha, j)}$-term in the series $\Psi_{\alpha}$ at the simple fraction with the pole $\hbar=-u_{j}(\alpha) / n$. The vector $d^{\prime}=$ $d+n d(\alpha, j)$ is automatically in $\Delta_{\alpha}^{*}$, and additionally $D_{j}+n \geq n$. Vice versa, when $d^{\prime}$ is in $\Delta_{\alpha}^{*}$, and for some $j \notin \alpha$ and $n \in \mathbb{N}$ we have $D_{j}^{\prime} \geq n$, then $d=d^{\prime}-n d(\alpha, j)$ is in $\Delta_{\beta(\alpha, j)}^{*}$ and $D_{j^{\prime}}+n=D_{j^{\prime}}^{\prime} \geq 0$.

Thus the sum of simple fractions on the RHS of the recursion relations reproduces all the simple fractions of the LHS with non-zero poles, and the remaining part of each $Z_{\alpha}^{d^{\prime}}(\hbar)$ is a Laurent polynomial of $\hbar$. Since the identity between the $q$-series with coefficients in $\mathbb{Q}\left(\hbar, \lambda, \lambda^{\prime}\right)$ holds for generic values of $\left(\lambda, \lambda^{\prime}\right)$ it holds in fact over $\mathbb{Q}\left(\hbar, \lambda, \lambda^{\prime}\right)$.

Remark. The coefficients $C_{\alpha, j}(n)$ in the recurrence relations of Propositions 4.4 and 6.3 are the same. Although we obtained this fact by straightforward computations of both coefficients, the coincidence should not be considered a miracle. The value of the coefficient $C_{\alpha, j}(n)$ in Proposition 4.4 is determined by localizations to fixed points represented by irreducible degree $n$ maps $\mathbb{C} P^{1} \rightarrow X$. On the other hand, the graph spaces differ from the toric map spaces only near stable maps of reducible curves. Perhaps the coincidence of the recurrence coefficients can be derived from this observation.

Consider now the case of complete intersections $Y$ with non-negative 1-st Chern class $c_{1}\left(\mathcal{T}_{Y}\right)$. This means that

$$
\sum_{i} p_{i}\left(\sum_{j} m_{i j}-\sum_{a} l_{i a}\right) \in \bar{K} .
$$

A reformulation of this condition reads: $\operatorname{deg} q^{d} \geq 0$ for all $d \in \Lambda$.

Put

$$
\begin{gathered}
\Lambda_{0}=\left\{d \in \Lambda \mid \sum L_{a}=\sum D_{j}, D_{j} \geq 0 \forall j=1, \ldots, N\right\}, \\
\Lambda_{1}=\left\{d \in \Lambda \mid \sum D_{j}-\sum L_{a}=1, D_{j} \geq 0 \forall j=1, \ldots, N\right\}, \\
\Lambda_{0}^{\prime}=\left\{d \in \Lambda \mid \sum L_{a}=\sum D_{j}\right\} .
\end{gathered}
$$


Proposition 6.4. Suppose that $\mathcal{T}_{Y}$ is non-negative. Then

$$
\begin{gathered}
\Psi=\Psi^{(0)}+\Psi^{(1)} / \hbar+o(1 / \hbar), \\
\Psi^{(0)}=\sum_{d \in \Lambda_{0}} \frac{L_{1} ! \ldots L_{l} !}{D_{1} ! \ldots D_{N} !} q^{d}, \\
\Psi^{(1)}=\sum_{d \in \Lambda_{1}} \frac{L_{1} ! \ldots L_{l} !}{D_{1} ! \ldots D_{N} !} q^{d}+\sum_{a} g_{a}(q) v_{a}-\sum_{j} f_{j}(q) u_{j},
\end{gathered}
$$

where $g_{a}$ and $f_{j}$ are some power series $\sum_{d \in \Lambda_{0}^{\prime}} A_{d} q^{d}$ with coefficients $A_{d} \in \mathbb{Q}$ and $A_{0}=0$.

Proof. In the case of non-negative $\mathcal{T}_{Y}$ we have $\sum D_{j} \geq \sum L_{a}$ for all $d \in \Lambda$. The definition of $\Psi\left(q, \hbar ; \lambda, \lambda^{\prime}\right)$ then shows that it can be written as a power series in $1 / \hbar$, and that the terms of order $\hbar^{0}$ and $\hbar^{-1}$ have the form described in the proposition.

7. Equivalence transformations. We study further the case of complete intersections $Y$ with non-negative tangent sheaf.

Consider a series $Z\left(q, \hbar ; \lambda, \lambda^{\prime}\right) \in H_{T}^{*}(Y, \mathbb{Q})$ of weighted-homogeneous degree 0 with respect to the grading $\operatorname{deg} \hbar=\operatorname{deg} \lambda=\operatorname{deg} \lambda^{\prime}=1, \operatorname{deg} q_{i}=$ $\sum_{j} m_{i j}-\sum_{a} l_{i a}$ which satisfies

(i) the recursion relation

$$
\begin{gathered}
Z_{\alpha}\left(q, \hbar ; \lambda, \lambda^{\prime}\right)=\sum_{d \in \Lambda} R_{\alpha, d}\left(\hbar^{-1} ; \lambda, \lambda^{\prime}\right) q^{d}+ \\
\sum_{j \notin \alpha} q^{n d_{\alpha, j}} \frac{C_{\alpha, j}(n)}{n \hbar+u_{j}(\alpha)} Z_{\beta(\alpha, j)}\left(q,-u_{j}(\alpha) / n ; \lambda, \lambda^{\prime}\right),
\end{gathered}
$$

where $R_{\alpha, d}$ are polynomials in $\hbar^{-1}$ with coefficients in $\mathbb{Q}\left(\lambda, \lambda^{\prime}\right)$ and the recursion coefficients $C_{\alpha, j}(n)$ are described in Propositions 4.4 and 6.3;

(ii) the condition that the series

$$
W=\sum w_{d, m} q^{d} z^{m}:=\int_{[Y]} Z\left(q e^{\hbar z}, \hbar\right) e^{p z} Z(q,-\hbar)
$$

has polynomial coefficients $w_{d, m} \in \mathbb{Q}\left[\hbar, \lambda, \lambda^{\prime}\right]$.

In Section 4 we proved that the series $\mathcal{S}$ is uniquely determined by these properties and the asymptotical condition of Corollary 4.3. In the previous 
section we found a series $\Psi$ which also has these properties but may differ from $\mathcal{S}$ by the asymptotical condition. In this section we describe some transformations of the series $Z$ which preserve the properties (i),(ii) but change the asymptotical expansion $Z=Z^{(0)}+Z^{(1)} / \hbar+o(1 / \hbar)$.

Proposition 7.1. Let $f=\sum_{\Lambda_{0}^{\prime}} f_{d} q^{d}$ be a series with rational coefficients $f_{d} \in \mathbb{Q}$ and $f_{0} \neq 0$. Then $f Z$ satisfies the conditions (i),(ii).

Proof. Simultaneous multiplication of the localizations $Z_{\alpha}$ by $f$ obviously preserves the the recursion relation of Proposition 4.4 and affects the series $\sum R_{\alpha, d} q^{d}$ in such a way that the polynomiality property of $R_{\alpha, d}(1 / \hbar)$ is preserved. This proves (i).

The multiplication of $Z$ by $f$ gives rise to the multiplication of the series $W$ by $f(q \exp (\hbar z)) f(q)$ which obviously preserves the polynomiality property of the coefficients $w_{d, m}$. This proves (ii).

Proposition 7.2. Let $f=\sum_{d \in \Lambda_{0}^{\prime} \cup \Lambda_{1}} f_{d} q^{d}$ be a series of weighted - homogeneous degree 1 where $f_{d}$ are linear inhomogeneous functions of $\lambda, \lambda^{\prime}$ with rational coefficients, and $f_{0}=0$. Then $\exp (f / \hbar) Z$ satisfies the conditions (i), (ii).

Proof. We have

$\exp \left(-n f / u_{j}(\alpha)-f / \hbar\right)=\exp \left(-\left(n \hbar+u_{j}(\alpha) f / u_{j}(\alpha) \hbar\right)=1+\left(n \hbar+u_{j}(\alpha)\right) g_{\alpha, j, n}\right.$,

where $g_{\alpha, j, n}$ is a $q$-series with coefficients polynomial in $\hbar^{-1}$ and rational in $\left(\lambda, \lambda^{\prime}\right)$. This implies that $\exp (f / h) Z_{\alpha}$ satisfy the recursion relation (i) with the new initial condition

$$
\begin{gathered}
e^{f / \hbar} \sum_{d \in \Lambda} R_{d, \alpha} q^{d}+ \\
e^{f / \hbar} \sum_{j \notin \alpha} \sum_{n=1}^{\infty} g_{\alpha, j, n} q^{n d_{\alpha, j}} C_{\alpha, j}(n) Z_{\beta(\alpha, j)}\left(q,-u_{j}(\alpha) / n ; \lambda, \lambda^{\prime}\right)
\end{gathered}
$$

whose coefficients at $q^{d}$ are still polynomial in $1 / \hbar$.

The transformation $Z \mapsto \exp (f / \hbar) Z$ multiplies the series $W$ by $\exp g$, where $g=\left(f\left(q e^{\hbar z}\right)-f(q)\right) / \hbar$ has polynomial coessicients when written as a power series in $(q, z)$ since $\exp (\hbar z d)-1$ is divisible by $\hbar$.

Proposition 7.3. Let the linear combination $f p=f_{1} p_{1}+\ldots+f_{k} p_{k}$ of the equivariant cohomology classes $p_{i} \in H_{T}^{*}(Y, \mathbb{Q})$ be given by the series 
$f_{i}=\sum_{d \in \Lambda_{0}^{\prime}} f_{i, d} q^{d}$ with rational coefficients $f_{i, d} \in \mathbb{Q}$ and $f_{i, 0}=0$. Then $\exp (f p / \hbar) Z\left(q \exp f, \hbar ; \lambda, \lambda^{\prime}\right)$ satisfies the conditions (i), (ii).

Proof. For $\beta=\beta(\alpha, j)$ we have $p(\alpha)-p(\beta)=d_{\alpha, j} u_{j}(\alpha)$ and therefore

$$
\begin{gathered}
\exp \left[-f p(\alpha) / \hbar-n f p(\beta) / u_{j}(\alpha)\right]= \\
e^{n d_{\alpha, j} f} \exp \left[-f p(\alpha)\left(n \hbar+u_{j}(\alpha)\right) /\left(u_{j}(\alpha) \hbar\right)\right]=e^{n d_{\alpha, j} f}+\left(n \hbar+u_{j}(\alpha)\right) g_{\alpha, j, n},
\end{gathered}
$$

where the series $g_{\alpha, j, n}\left(q, \hbar^{-1} ; \lambda, \lambda^{\prime}\right)$ has the same properties as specified in the proof of Proposition 7.2. This implies that the recursion relation for $e^{f p / \hbar} Z\left(q e^{f}, \hbar\right)$ (with some initial conditions $\sum R_{\alpha, d} q^{d}$ ) follows from the recursion relation obtained by the change $q \mapsto q e^{f}$ from (i) satisfied by $Z$.

The operation $Z \mapsto e^{f p} Z\left(q e^{f}, \hbar\right)$ transforms $W(z, q)$ to

$$
W\left(z+\frac{f\left(q e^{\hbar z}\right)-f(q)}{\hbar}, q e^{f}\right)
$$

and preserves the polynomiality property of the coefficients $w_{d, m}$ since coefficients of the series $f_{i}\left(q e^{\hbar z}\right)-f_{i}(q)$ are divisible by $\hbar$.

Using the notations from Proposition 6.4 we perform the following operations with the series $\Psi$ which according to Propositions 7.1, 7.2, 7.3 preserve the properties (i),(ii).

(1) Divide $\Psi$ by $\Psi^{(0)}$; this transforms the asymptotical expansion $\Psi=$ $\Psi^{(0)}+\Psi^{(1)} / \hbar+o(1 / \hbar)$ to

$$
1+\hbar^{-1}\left(H(q)+\sum_{a} G_{a}(q) v_{a}-\sum_{j} F_{j}(q) u_{j}\right)+o\left(\hbar^{-1}\right),
$$

where $H=\left(\sum_{d \in \Lambda_{1}} \frac{L_{1} ! \ldots L_{l} !}{D_{1} ! \ldots D_{N} !} q^{d}\right) / \Psi^{(0)}$, and $G_{a}=g_{a} / \Psi^{(0)}, F_{j}=f_{j} / \Psi^{(0)}$.

(2) Divide $\Psi / \Psi^{(0)}$ by $\exp \hbar^{-1}\left(H+\sum_{j} F_{j} \lambda_{j}-\sum_{a} G_{a} \lambda_{a}^{\prime}\right)$; this transforms the asymptotical expansion to

$$
Z=1+\hbar^{-1} \sum_{i} \phi_{i} p_{i}+o\left(\hbar^{-1}\right)
$$

where $\phi_{i}=\sum_{a} l_{i a} G_{a}-\sum_{j} m_{i j} F_{j}$.

(3) Transform $\exp \left(-\sum \phi_{i} p_{i} / \hbar\right) Z(q, \hbar)$ to the new variables $Q_{i}=q_{i} e^{\phi_{i}(q)}$, $i=1, \ldots, k$; the resulting series $S\left(Q, \hbar ; \lambda, \lambda^{\prime}\right)$ has the asymptotical expansion $1+o(1 / h)$. 
Corollary 7.4. Suppose that $\mathcal{T}_{Y}$ is non-negative. Then $\mathcal{S}\left(q, \hbar ; \lambda, \lambda^{\prime}\right)=$ $S\left(q, \hbar ; \lambda, \lambda^{\prime}\right)$.

Proof. The series $S$ satisfies the conditions (a),(b),(c) of Proposition 4.5 which uniquely determine the series $\mathcal{S}$.

\section{References}

[1] M. Audin, The topology of torus actions on symplectic manifolds. Birkhäuser, Basel, 1991.

[2] V. Batyrev, Dual polyhedra and mirror symmetry for Calabi-Yau hypersurfaces in toric varieties. J. Alg. Geom. 3 (1994), 493 - 535.

[3] V. Batyrev, Quantum cohomology rings of toric manifolds. Preprint, alg-geom/9310004.

[4] V. Batyrev, D. van Straten, Generalized hypergeometric functions and rational curves on Calabi-Yau complete intersections in toric varieties. Comm. Math. Phys. 168 (1995), 493 - 533.

[5] K. Behrend, Yu. Manin, Stacks of stable maps and Gromov - Witten invariants. Duke Math. J. 85 (1996), 1 - 60.

[6] T. Eguchi, K. Hori, C.-S. Xiong, Gravitational quantum cohomology. Preprint, 1996.

[7] A. Givental, A symplectic fixed point theorem for toric manifolds. Preprint UC Berkeley, 1992. Published in: The Floer memorial volume. H. Hofer, C. H. Taubs, A. Weinstein, E. Zehnder (eds.), Progress in Math. 133, Birkhäuser, 1995, $445-481$.

[8] A. Givental, Homological geometry I: projective hypersurfaces. Selecta Math., New series, v.1, No. 2, $325-345$.

[9] A. Givental, Homological geometry and mirror symmetry. In: Proceedings of the ICM, Zürich 1994, Birkhäuser, 1995, v. 1, $472-480$.

[10] A. Givental, Equivariant Gromov - Witten invariants. IMRN, 1996, No. 13, 613 - 663 .

[11] A. Givental, Stationary phase integrals, quantum Toda lattices, flag manifolds and the mirror conjecture. Preprint, alg-geom/9612001. 
[12] M. Kontsevich, Enumeration of rational curves via toric actions. In: The moduli spaces of curves, R. Dijkgraaf, C. Faber, G. van der Geer (eds.), Progress in Math. 129, Birkhäuser, 1995, 335 - 368.

[13] M. Kontsevich, Yu. Manin, Gromov - Witten classes, quantum cohomology and enumerative geometry. Comm. Math. Phys. 164 (1994), 525 -562 .

[14] J. Li, G. Tian, Virtual moduli cycles and Gromov - Witten invariants of algebraic varieties. Preprint, alg-geom/9602007.

[15] J. Li, G. Tian, Virtual moduli cycles and Gromov - Witten invariants in general symplectic manifolds. Preprint, alg-geom/9608032.

[16] D. Morrison, R. Plesser, Summing the instantons: Quantum cohomology and mirror symmetry in toric varieties. Nucl. Phys. B440 (1995), 279 - 354 (hep-th / 9412236).

[17] A. Schwarz, Sigma-models having supermanifolds as target spaces. Preprint, IHES, 1995. 